2019-05-08

\title{
Smoothed particle hydrodynamics A
} new approach for modeling flow in oscillatory baffled reactors

\author{
Jimeno, G
}

http://hdl.handle.net/10026.1/17099

10.1016/j.compchemeng.2019.02.003

Computers and Chemical Engineering

Elsevier

All content in PEARL is protected by copyright law. Author manuscripts are made available in accordance with publisher policies. Please cite only the published version using the details provided on the item record or document. In the absence of an open licence (e.g. Creative Commons), permissions for further reuse of content should be sought from the publisher or author. 


\section{Accepted Manuscript}

Smoothed Particle Hydrodynamics - A New Approach for Modeling Flow in Oscillatory Baffled Reactors

Guillermo Jimeno, Yeaw Chu Lee, Xiong-Wei Ni

PII: S0098-1354(18)30745-2

DOI: https://doi.org/10.1016/j.compchemeng.2019.02.003

Reference: CACE 6341

To appear in: Computers and Chemical Engineering

Received date: 17 July 2018

Revised date: 24 January 2019

Accepted date: 1 February 2019

Please cite this article as: Guillermo Jimeno, Yeaw Chu Lee, Xiong-Wei Ni, Smoothed Particle Hydrodynamics - A New Approach for Modeling Flow in Oscillatory Baffled Reactors, Computers and Chemical Engineering (2019), doi: https://doi.org/10.1016/j.compchemeng.2019.02.003

This is a PDF file of an unedited manuscript that has been accepted for publication. As a service to our customers we are providing this early version of the manuscript. The manuscript will undergo copyediting, typesetting, and review of the resulting proof before it is published in its final form. Please note that during the production process errors may be discovered which could affect the content, and all legal disclaimers that apply to the journal pertain. 


\section{HIGHLIGHTS}

- For the first time, Smoothed-Particle Hydrodynamics (SPH) has been implemented to predict and assess mixing behaviors in oscillatory baffled reactors.

- Velocity fields and flow patterns modelled by SPH match closely to those by its Eulerian counterpart, i.e. Finite Volume (FV) method.

- Historical information of individually tracked fluid particles, provided by SPH, is directly applied to quantify mixing performance using the existing velocity ratio and stretch rate indexes.

- By making full use of SPH's capabilities, two new indexes are proposed for the quantification of mixing and plug flow efficiency of the device. 


\title{
Smoothed Particle Hydrodynamics - A New Approach for Modeling Flow in Oscillatory Baffled Reactors
}

\author{
Guillermo Jimeno, Yeaw Chu Lee and Xiong-Wei Ni*
}

EPSRC, Centre for Continuous Manufacturing and Crystallisation (CMAC), Centre for Oscillatory Baffled Reactor Applications (COBRA), School of Engineering and Physical Science, Heriot Watt University, Edinburgh. EH144AS, UK

*Corresponding author; email: x.ni@hw.ac.uk; Tel; 00441314513781

\begin{abstract}
Existing numerical models of mixing processes in oscillatory baffled reactors (OBR) are mainly Eulerian-based. An alternative Lagrangian based methodology, Smoothed-Particle Hydrodynamics (SPH), for predicting flow patterns and assessing mixing performance is presented in this paper. A bespoke SPH solver is developed for single phase modelling, as it is, for the first time, applied to OBR, and the results are compared with those from Eulerian modelling, i.e. Finite Volume (FV) method. SPH has successfully captured the expected flow characteristics in OBR as clearly and equally as its Eulerian counterpart, validating the SPH method. Since SPH provides historical information of individually tracked fluid packets/particles in the domain of interest, it allows for readily quantitative assessments of mixing without additional models. Two new indexes to assess mixing and plug flow efficiency have been proposed by making full use of SPH's capabilities.
\end{abstract}


Key words: Smoothed Particle Hydrodynamics (SPH); Computational Fluid Dynamics (CFD); Lagrangian based modeling; oscillatory baffled reactor; flow patterns; mixing efficiency

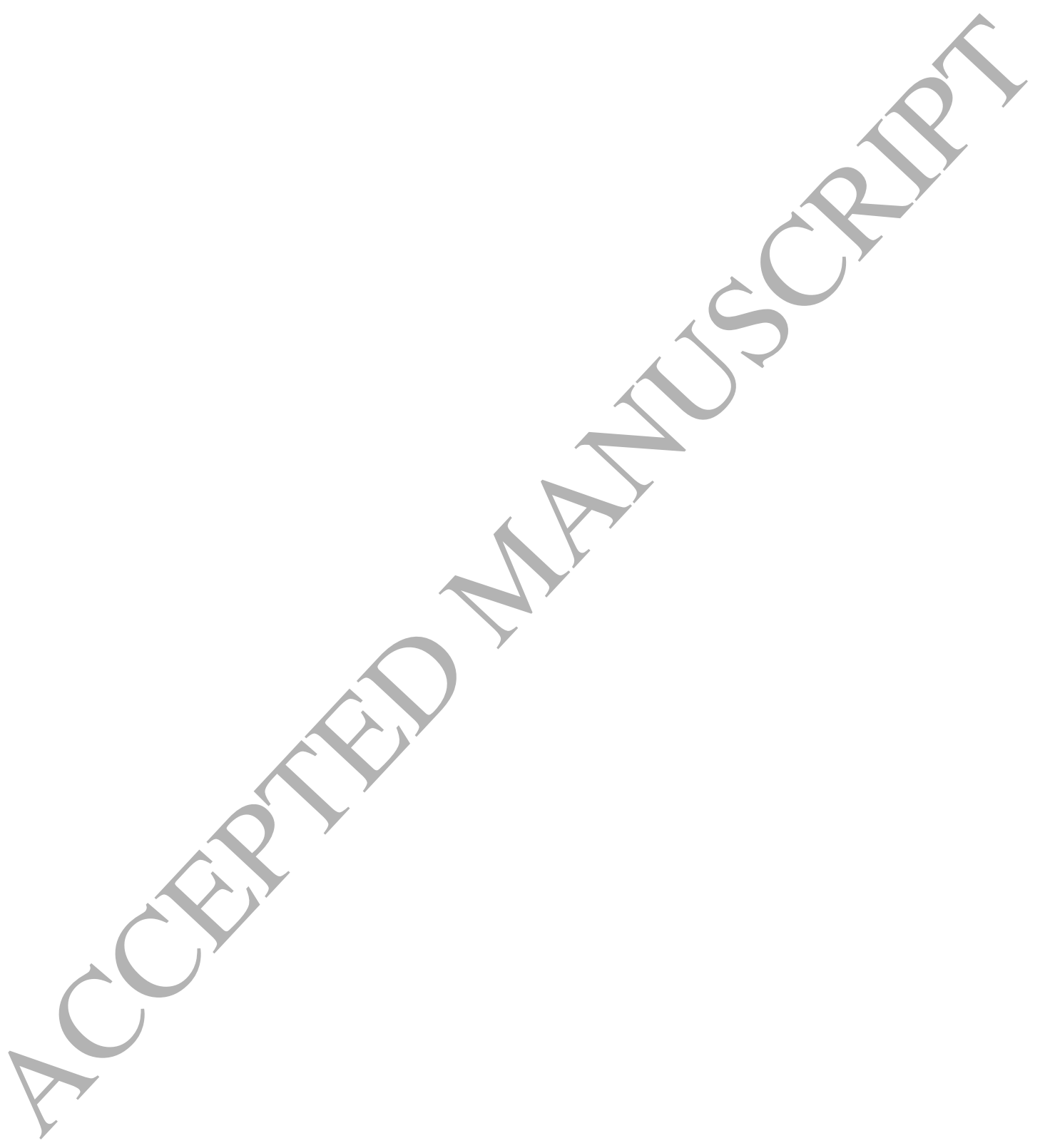




\section{Introduction}

Uniform mixing and linear scale up offered by Oscillatory Baffled Reactors (OBR) are the key drivers for research and industrial applications in reactions (Eze et al., 2017; Navarro Fuentes et al., 2018; Ni et al., 1999; Phan et al., 2012; Wilson et al., 2001) and crystallization (Agnew et al., 2017; Brown et al., 2015; Brown et al., 2018; Brown \& Ni, 2012; Brown \& Ni, 2011; Callahan \& Ni, 2012; Feilden, 2017; Lawton et al., 2009; Ni et al., 2004; Su et al., 2015). From a modelling viewpoint, Computational Fluid Dynamic (CFD) solvers have been employed to simulate hydrodynamic flow profiles in OBR from initially 2 -dimensional in the 1990s (Howes et al., 1991; Howes \& Shardlow, 1997; Mackley \& Neves Saraiva, 1999; Mackley \& Roberts, 1991; Roberts, 1994; Roberts \& Mackley, 1995, 1996) to 3-D in the following decades (Chew et al., 2004; Deng et al., 2010; Fitch et al., 2005; Hamzah et al., 2012; Jian \& Ni, 2003; Jian \& Ni, 2005; Mazubert et al., 2016a; Ni et al., 2003; Ni et al., 2002; Nogueira et al., 2013; Reis et al., 2005; Reis et al., 2010; Zheng et al., 2007), however the majority of the numerical modelling work was Eulerian based (González-Juárez et al., 2017; Manninen et al., 2013) mostly for single phase. The validation of these numerical models with experimental data has mostly been limited to qualitative comparison of eddy formation patterns. There were few modelling work involving two phases, e.g. solids and liquid (Mazubert et a1., 2016b), which is largely limited by the expensive computational costs of coupling continuous Eulerian-Lagrangian phases for dynamic fluid-solids modelling and their complex/interactions. Coupling CFD with the Population Balance Equation (PBE) to model crystallization processes in stirred tank crystallizers is a well-documented example (Yang \& Mao, 2014). The question remains if the complexity of these coupled methodologies can be avoided and if all phases can be modeled using a Lagrangian scheme; the SmoothedParticle Hydrodynamics ( $\mathrm{SPH}$ ) is thus the one considered in the current study. The SPH methodology provides historical information of individual fluid packets or particles in the 
domain of interest by tracking these particles; this implicit SPH capability opens up opportunities for better understanding of flow rheology behavior. This is of special interest in complex processes where L-L and S-L interactions play a key role, such as the chemical processes commonly undertaken in OBRs, e.g. crystallization. This can greatly improve the accuracy in the prediction of mixing, since historical information of particles allows for alternative ways to quantify mixing, as opposed to residence time distributions and axial dispersion assessments traditionally used in OBRs. The quantification of mixing efficiency in OBRs is of great interest and has been a hot topic of research since the 1990s in both experimental (Mackley \& Ni, 1993; Ni, 1994; Ni, 1995; Ni \& Stevenson, 1999; Palma \& Giudici, 2003; Phan \& Harvey, 2010; Phan \& Harvey, 2011; Reis et al., 2004; Zheng \& Mackley, 2008) and numerical studies (González-Juáréz et al., 2018; González-Juárez et al., 2017; Howes et al., 1991; Jian \& Ni, 2003; Kimuli et al., 2017; Manninen et al., 2013; Reis et al., 2010), since both uniform mixing and heat transfer control in OBRs are the essential elements for achieving consistent product properties in these undertaken processes, e.g. narrow crystal size distribution, constant polymorph. In addition, SPH allows for the implementation of new physics based on particle-particle interaction, which has great potential for modelling solids formation and growth in crystallization processes.

SPH was first developed in 1977 for astrophysical applications by Gingold and Monaghan (Gingold \& Monaghan, 1977) and its application into fluid flow problems was proposed by Monaghan in the early 1990s (Monaghan, 1992). This methodology, despite being less popular than traditional standard CFD approaches, is well documented (Violeau, 2012), and has augmented vast research attention in recent years in solving complex fluid flow problems (Adami et al., 2013; Crespo et al., 2007; Dalrymple \& Rogers, 2006; Ferrari et al., 2009; Gomez-Gesteira et al., 2010; Zhang et al., 2017) in micro and macro-scales. To the authors' 
knowledge there has been no reported work on the application of SPH for modelling fluid flows in tubular baffled reactors. The aims of this work are to explore and investigate the feasibility of SPH in OBR by developing a bespoke solver; to compare flow characteristics of single phase flow predicted by SPH with those obtained with an Eulerian based model, e.g. Finite Volume (FV), via a commercial software package (ANSYS® Fluent 15) and to provide quantitative assessment of mixing efficiency using the proposed methodology.

\section{Problem definition}

Oscillatory baffled reactors are tubular devices that contain periodically spaced orifice baffles with superimposed fluid oscillation; mixing is generated by the formation and cessation of eddies, providing uniform mixing in each inter-baffled section(Jian et al., 2004; Mackley \& $\mathrm{Ni}, 1991)$. The sinusoidal oscillatory inlet flow is imposed by a piston at the entrance of the reactor. The displacement of the piston and the oscillatory inlet velocity of the reactor are represented by:

$$
x_{p}(t)=-x_{o} \cos (\omega t)
$$

$$
u_{\text {inlet }}(t)=\omega x_{o} \sin (\omega t)
$$

where $x_{p}$ is the piston's position $(\mathrm{m}), u_{\text {inlet }}$ the inlet mean velocity $\left(\mathrm{m} \mathrm{s}^{-1}\right), \omega=2 \pi f$ the oscillation angular frequency $\left(\mathrm{rad} \mathrm{s}^{-1}\right), f$ the oscillation frequency $(\mathrm{Hz}), x_{o}$ the oscillation center-to-peak amplitude (m) and $t$ the time (s). Figure 1 and Table 1 show the schematic and geometry of the oscillatory baffled reactor system. 


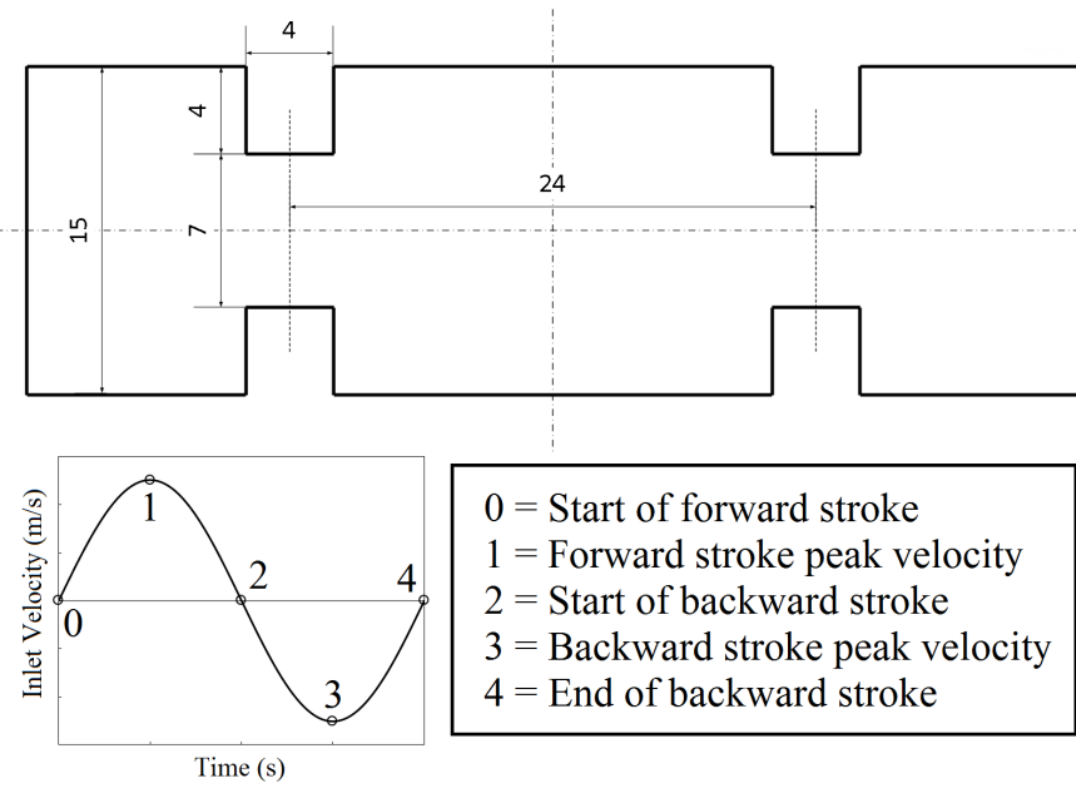

Figure 1. Schematic of the baffled cell and inlet velocity profile in OBR; all quotes are in $\mathrm{mm}$

Table 1. Geometry dimensions of the OBR

\begin{tabular}{lr}
\hline$D(\mathrm{~mm})$ & 15 \\
$D_{b}(\mathrm{~mm})$ & 7 \\
$L_{b}(\mathrm{~mm})$ & 24 \\
$L(\mathrm{~mm})$ & 48 \\
Baffled cells in reactor $(\#)$ & 2 \\
\hline
\end{tabular}

where $D$ is the diameter of the tube $(\mathrm{m}), D_{b}$ the diameter of baffle hole $(\mathrm{m}), L_{b}$ the baffle spacing $(\mathrm{m})$ and $L$ the length of the reactor $(\mathrm{m})$. Water is used as the working fluid with oscillatory conditions of $x_{o}=5 \mathrm{~mm}$ and $f=1 \mathrm{~Hz}\left(R e_{o}=\omega x_{o} \rho D / \mu=471\right.$ and $S t=D /\left(4 \pi x_{o}\right)=$ 0.239); these conditions are chosen to avoid interactions among eddies generated during the forward and backward strokes so that symmetrical flow patterns are expected. 


\section{Numerical formulation}

The simulated system comprises a 2-dimensional domain and it is assumed iso-thermal. Navier-Stokes equations for mass and momentum conservation are solved according to:

$$
\frac{D \rho}{D t}+\rho \nabla \cdot \vec{u}=0
$$

$$
\frac{D(\rho \vec{u})}{D t}=\nabla \cdot \underline{\underline{\sigma}}+\rho \vec{g}+\sum \vec{F}
$$

where $\frac{D}{D t}$ is the material derivative, $\nabla$ the gradient vector operator, $\rho$ is the density, $\vec{u}$ the velocity vector, $\underline{\underline{\sigma}}$ the total stress tensor defined as $\underline{\underline{\sigma}}=-p \underline{\underline{I}}+\underline{\underline{\tau}}$ and $\sum \vec{F}$ the summation of any external forces. Within the stress tensor, $\underline{\underline{I}}$ is the identity matrix, $p$ the pressure, $\underline{\underline{\tau}}=\left(-\frac{2}{3} \mu \nabla \cdot \vec{u}\right) \underline{\underline{I}}+2 \mu \underline{\underline{E}}$ is the stress tensor with $\underline{\underline{E}}$, the strain rate of tensor, given by $\underline{\underline{E}}=\frac{1}{2}\left[(\nabla \vec{u})+(\nabla \vec{u})^{T}\right]$. Equations (3) and (4) are discretized and solved separated using SPH and FV in their respective solvers. Note that the governing equations have been solved in a 2dimensional form, neither for FV nor SPH were these solved axisymmetrically.

\subsection{Smoothed-Particle Hydrodynamic}

SPH discretizes the fluid domain using a fixed number of infinitesimal particles that are tracked individually. Assuming that the system is fully flooded with a single-phase fluid, no external forces are considered; hence Equations (3) and (4) can be written in their Lagrangian form as: 


$$
\frac{D \rho}{D t}=-\rho \nabla \cdot \vec{u}
$$

$$
\frac{D \vec{u}}{D t}=-\frac{1}{\rho} \nabla \cdot p \underline{\underline{I}}+\frac{1}{\rho} \nabla \cdot \underline{\underline{\tau}}
$$

and the position of each infinitesimal particle $\vec{r}$ is governed by:

$$
\frac{d \vec{r}}{d t}=\vec{u}
$$

This system of equations is closed using the relationship between density and pressure. Here, the fluid domain is treated as weakly compressible, for which this relationship is given by the Equation-Of-State (EOS) (Monaghan, 1992). The most common EOS was proposed by Batchelor (Batchelor, 1974), the so-called Tait's equation:

$$
p=B\left[\left(\frac{\rho}{\rho_{0}}\right)^{\gamma}-1\right]
$$

where $\gamma$ usually takes a value of 7 for water, $\rho_{0}$ is the reference density of the fluid at atmospheric pressure and the constant $B$ is defined as $B=\frac{\rho_{0} c_{0}^{2}}{\gamma} ; c_{0}$ is the reference speed of sound. The use of Tait's equation as EOS highly reduces computational time, as oppose to solving the Poisson's equation for incompressible flows (Lee et al., 2007). The compressibility of the fluid in the SPH's weakly compressible approach is controlled by $c_{o}$, which also determines the size of the time-step based on the Courant-Friedrichs-Lewy condition (Courant et al., 1928), refer to Equation (15). High values of speed of sound result 
in both incompressible behavior and very small time-steps. However, Monaghan (Monaghan, 1994) demonstrated that $c_{0}$ values of ten to a hundred times the maximum velocity in the system, corresponding to a Mach number of less than 0.1, replicate incompressible flow with density fluctuations within $1 \%$. A value of $c_{0}=10 \mathrm{~m} \mathrm{~s}^{-1}$ was then chosen for the simulations performed in this study.

\subsubsection{Discretization and kernel approximation}

SPH treats a continuous medium as a discrete set of particles. At time zero, particles are uniformly distributed and are equidistantly spaced from each other, i.e. a distance $\Delta x$ in all directions. Hence, all particles have an initial volume of $\Delta x^{n}$ where $n$ is the dimensions of the domain. The discretization of Equations (5) and (6) is derived from the Dirac distribution $\delta$. Thus, the value of a function at a certain time, $t$, and at an arbitrary point or particle, $i$, can then be re-written as the summation the contributions from its neighboring particles, $j$ :

$$
f\left(\vec{r}_{i}, t\right) \approx \sum_{j=1}^{N} \frac{m_{j}}{\rho_{j}} f\left(\vec{r}_{j}, t\right) W\left(\vec{r}_{i}-\vec{r}_{j}, h\right)
$$

where $f$ is a function of the position vectors $\vec{r}_{i}$ and $\vec{r}_{j}$ - the former refers to the particle of interestes and the latter to each of its neighouring particles. The contribution of each neighboring particle is weighted by the use of a kernel function, $W$, based on the distance between particles $i$ and $j$; $h$ is the smoothing length of the chosen kernel function defined as $h=\eta \Delta x ; \eta$ typically takes a value of $1.2-1.3$, the latter is chosen for this work. From this point onwards, for simplicity purposes $W\left(\vec{r}_{i}-\vec{r}_{j}, h\right)$ will be denoted as $W_{i j}$ and $\left(\vec{r}_{i}-\vec{r}_{j}\right)$ as $\vec{r}_{i j}$; $N$ is the number of neighboring particles within the kernel domain. 
The kernel function $W_{i j}$ for smoothing length $h$ depends on the magnitude of the position vector $\vec{r}_{i j}$ as:

$$
W_{i j}=\frac{\alpha_{w, n}}{h^{n}} f(q)
$$

where $\alpha_{w, n}$ is the kernel renormalization term that depends on the kernel and dimensionality of the problem, $f(q)$ represents the kernel function that is a positive, symmetric and at least once continuously derivable function with $q=\frac{\left|\vec{r}_{i j}\right|}{h}$. In this study, the Wendland kernel is employed, as it provides a high order of interpolation with reasonable computational cost (Gomez-Gesteira et al., 2010); a high order of interpolation is required to capture complex fluid flow phenomena, leading to high degrees of accuracy and stability of the SPH scheme (Morris, 1996). The Wendland kernel (Wendland, 1995) is defined as:

$$
f(q)= \begin{cases}\left(1-\frac{q}{2}\right)^{4}(1+2 q) & 0 \leq q \leq 2 \\ 0 & 2<q\end{cases}
$$

where $\alpha_{w, 1}=\frac{3}{4}, \alpha_{y, 2}=\frac{7}{4 \pi}, \alpha_{w, 3}=\frac{21}{16 \pi}$ for 1,2 and 3 dimensions respectively.

\subsubsection{Continuity equation}

The continuity equation, in its SPH formulation, given in Equation (5), is then discretized and re-written as (Gingold \& Monaghan, 1977): 


$$
\frac{D \rho_{i}}{D t}=\sum_{j=1}^{N} m_{j} \vec{u}_{i j} \cdot \nabla_{i} W_{i j}
$$

where $i$ is the interested particle, $j$ any neighboring particle within the kernel domain, i.e. $\left|\vec{r}_{i j}\right|<2 h$ for the Wendland kernel, $\nabla_{i}$ the gradient of the kernel function with respect to the position vector $\vec{r}_{i j}$ and $\vec{u}_{i j}=\vec{u}_{i}-\vec{u}_{j}$ is the relative velocity between the particles.

\subsubsection{Momentum equation}

The momentum equation, in its SPH formulation, given in Equation (6), is discretized following the discretized form proposed by Morris (Morris et al., 1997):

$$
\frac{D \vec{u}_{i}}{D t}=-\sum_{j=1}^{N} m_{j}\left(\frac{p_{j}}{\rho_{j}^{2}}+\frac{p_{i}}{\rho_{i}^{2}}\right) \nabla_{i} W_{i j}+\sum_{j=1}^{N} m_{j}\left(\frac{\mu_{i}+\mu_{j}}{\rho_{i} \rho_{j}}\right) \vec{u}_{i j}\left(\frac{1}{\left|\vec{r}_{i j}\right|} \frac{\partial W_{i j}}{\partial\left|\vec{r}_{i j}\right|}\right)
$$

\subsubsection{Density-Smoothing method}

Equations (12) and (13) are solved and regarded as the standard weakly compressible SPH method. However, while kinematics in SPH is well understood, the weakly compressible approach can result in pressure fluctuations between particles, translating into numerical noise in the velocity field, which may exacerbate disorder and erratic motions of the fluid particles. One of the most straightforward and computationally least expensive approaches to tackle this issue is to perform a smoothing filter over the density of the particles (GomezGesteira et al., 2010). In the past, this has commonly been done by re-assigning a reference density value to each particle at set time intervals (Belytschko et al., 1998; Colagrossi \& Landrini, 2003; Dilts, 1999). A more elegant approach, utilized in this work, is to implement 
a simple density-smoothing model, analogous to the $\alpha-\mathrm{XSPH}$ model as proposed by Violeau (Violeau, 2012):

$$
\hat{\rho}_{i}=\rho_{i}-\varepsilon \sum_{j=1}^{N} m_{j} \frac{\rho_{i j}}{\bar{\rho}_{i j}} W_{i j}
$$

where $\varepsilon$ is a dimensionless coefficient, $\rho_{i j}=\rho_{i}-\rho_{j}$ and $\bar{\rho}_{i j}$ is the harmonic average. Values of the order of $10^{-2}$ are often recommended for the constant $\varepsilon$ of the density-smoothing function (Violeau, 2012); $\varepsilon=0.01$ is chosen in the present study. When this densitysmoothing method is implemented, Equation (14) is solved immediately after solving Equation (12).

\subsubsection{Time integration}

An adaptive time-stepping algorithm is used for calculating time-steps $\Delta t$. Here, three criteria are used which include the Courant-Friedrichs-Lewy condition (Courant et al., 1928):

$$
\Delta t_{C F L} \leq C_{C F L} \frac{h}{c_{0}}
$$

a constrain based on the force per unit mass of each particle (Monaghan, 1992), which is essentially the magnitude of particle acceleration $\left|\vec{f}_{i}\right|$ :

$$
\Delta t_{\text {force }} \leq C_{\text {force }} \min _{i}\left(\sqrt{\frac{h}{\left|\vec{f}_{i}\right|}}\right)
$$

and an additional constrain due to viscous diffusion (Morris et al., 1997): 


$$
\Delta t_{v i s c} \leq C_{v i s c} \min _{i}\left(\frac{h^{2}}{v_{i}}\right)
$$

where the kinematic viscosity $v_{i}=\mu_{0} / \rho_{i}$ and $\mu_{0}$ is the reference viscosity of the fluid. Values for $C_{C F L}, C_{f o r c e}$ and $C_{\text {visc }}$ of $0.01,0.0125$ and 0.0125 , respectively, are conservatively chosen to ensure stability of the solution. The final time-step was chosen as the minimum of these three conditions:

$$
\Delta t \leq \min \left(\Delta t_{C F L}, \Delta t_{\text {force }}, \Delta t_{\text {visc }}\right)
$$

Time was integrated explicitly using the second order accurate Verlet algorithm (Verlet, 1967):

$\rho_{i, n+1}=\rho_{i, n-1}+\left(\Delta t_{n-1}+\Delta t_{n}\right)\left(\frac{D \rho_{i}}{D t}\right)$

$$
\vec{u}_{i, n+1}=\vec{u}_{i, n-1}+\left(\Delta t_{n-1}+\Delta t_{n}\right)\left(\frac{D \vec{u}_{i}}{D t}\right)_{n}
$$

$$
\vec{r}_{i, n+1}=\vec{r}_{i, n}+\vec{u}_{i, n} \Delta t t_{n}+\frac{\Delta t_{n}^{2}}{2}\left(\frac{D \vec{u}_{i}}{D t}\right)_{n}
$$

where the past, current and future temporal steps are represented by $n-1, n$ and $n+1$, respectively. Note that the use of Equations (19) and (20) will eventually lead to a decoupled system. In order to prevent the solution at odd and even time-steps from diverging, an Euler upwind integration is performed at every $M$ time-steps; a value of $M$ of 50 is presently used. 


\subsubsection{Boundary conditions}

The walls that define the limits of the fluid domain were simulated using dynamic boundary conditions (Crespo et al., 2007), chosen for its computational simplicity. Particles comprising dynamic boundaries are solved like any other fluid particles following the solution of Equations (8), (12) and (13). However, the particle positions remain fixed and are not updated in subsequent iterations in time. In order to ensure all fluid particles have consistently the same number of neighboring particles at all times, solid wall boundaries were modeled with three consecutive rows of dynamic particles.

In order to model the oscillatory behavior of the fluid, two pistons, one on the left and another one on the right, are defined on both ends of the OBR, as shown in Figure 2, using dynamic particles whose positions and velocities are controlled by Equations (1) and (2).

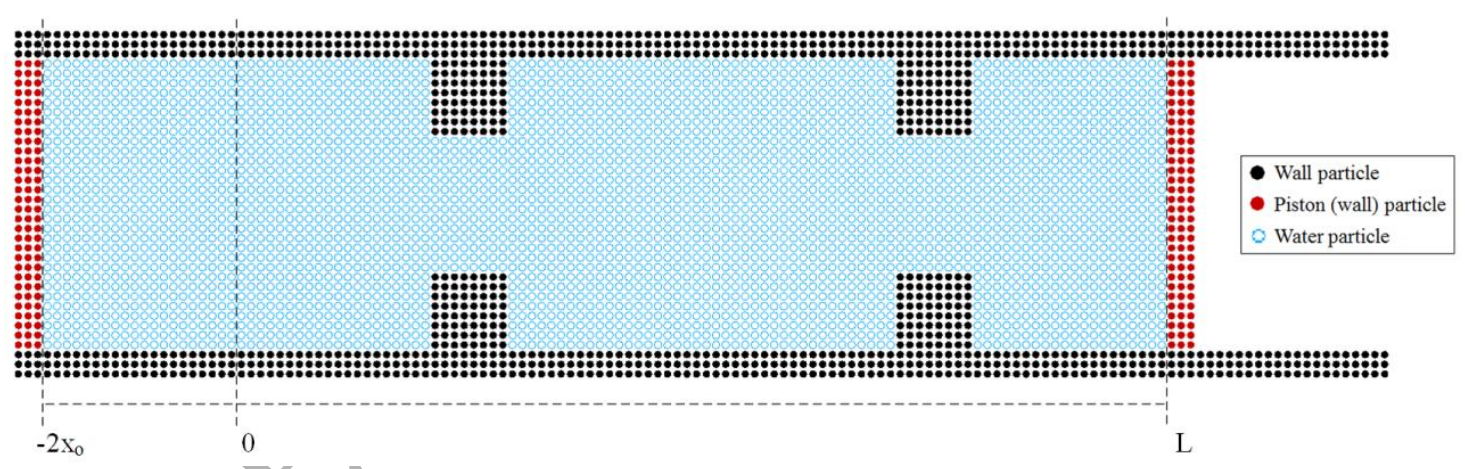

Figure 2. SPH OBR model for $\triangle x=0.0005 m$

\subsubsection{Post-processing monitors}

The velocity profiles of the flow in the OBR are evaluated along three different lines as depicted in Figure 3: a vertical line at the middle of the left baffle (Line 1) - the section experiencing the highest velocity; a centered vertical line (Line 2) - the area where the 
strongest eddy dissipation occurs; and a center horizontal line (Line 3) - the area with the weakest eddy interaction as aforementioned. The velocity magnitude $u_{m}$ at each point of a line, defined at equidistance intervals of $\Delta x$ along the monitor lines, is calculated analogously to Equation (9), given by:

$$
u_{m, i}=\sum_{j=1}^{N} \frac{m_{j}}{\rho_{j}} u_{m, i} W_{i j}
$$

where $i$ is the interpolated monitor point that utilizes information from surrounding particles within the kernel smoothing length.

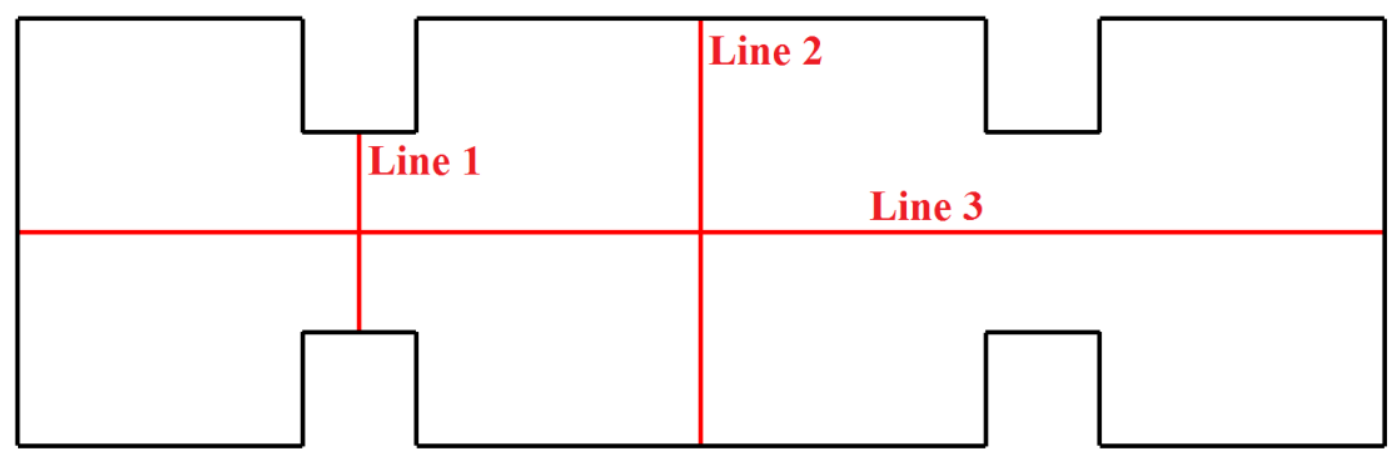

Figure 3. Position of lines at which results are extracted and evaluated

\subsection{Finite volume method}

For the purpose of comparison, the Eulerian based Finite Volume (FV) method is also applied to the same geometry and operational conditions as given in Table 1 and Figure 1 . The following Navier Stokes for continuity and momentum conservation equations, given by Equations (3) and (4), are simplified and solved for incompressible flow: 
$\nabla \cdot \vec{u}=0$

$\rho\left(\frac{\partial \vec{u}}{\partial t}+\vec{u} \cdot \nabla \vec{u}\right)=-\nabla p+\mu \nabla^{2} \vec{u}$

\subsubsection{Numerical Setup}

All the numerical FV modelling is performed using the ANSYS® Fluent 15.0 CFD package. Simulations are done by the pressure-based segregated solver, using the SIMPLE pressurevelocity coupling algorithm. A second order upwind scheme was utilized for the spatial discretization of the momentum equation; a second order scheme for the interpolation of pressure at the faces of the grid and a second order implicit scheme for time discretization. The time-step employed is 0.001 seconds throughout all simulations and the convergence criteria is set to residuals of $10^{-5}$ for solving both Equations (23) and (24) to ensure accuracy. The average value of the Courant-Friedrichs-Lewy (CFL) coefficient was kept below 0.6 and the maximum CLF value below 4.1.

To replicate the OBR with the double piston in the SPH approach, flat velocity profiles are defined on both ends of the OBR using a customized User Defined Function (UDF) in ANSYS® Fluent given by Equation (2); walls were model as no-slip boundaries. Equations (23) and (24) were solved as laminar, i.e. no turbulence model was implemented. This is in agreement with literature, where laminar solver has been the norm for simulating flows in oscillatory baffled reactors in the past (Fitch et al., 2005; Ni et al., 2002; Reis et al., 2005) and present decade (González-Juárez et al., 2018; González-Juárez et al., 2017; Kimuli et al., 2017; Mazubert et al., 2016a, 2016b) under a FV framework, including flows with $\operatorname{Re}_{\mathrm{o}}$ values up to 8043 (Jian \& Ni, 2005; Manninen et al., 2013). 


\subsubsection{Mesh}

A mesh sensitivity analysis is performed using a selection of computational nodes per baffled cell. Meshes of the two baffled-cell domain of five different resolutions are analyzed for ten oscillatory cycles. Profiles of velocity magnitude extracted at Lines 1, 2 and 3, as shown in Figure 3, are then cycle-averaged over all ten oscillatory cycles and compared for strokes 1 to 4 (refer to Figure 1) for various mesh densities. The coefficient of determination, $\mathrm{R}^{2}$, was selected for comparison between meshes, defined by:

$$
R^{2}=1-\frac{\sum_{b=1}^{k}\left(\phi_{a, b}-\phi_{1, \mathrm{~b}}\right)^{2}}{\sum_{b=1}^{k}\left(\phi_{a, b}-\overline{\phi_{1, \mathrm{~b}}}\right)^{2}}
$$

where $b$ and $k$ are, respectively, a single data point and the total number of data points of a profile, $a$ is the index of a certain mesh and $\phi$ the property under evaluation. A summary of the mesh independency analysis is shown in Table 2, clearly indicating that the resolution of mesh \#2 is the desired choice on the balance between accuracy and computation time, which is selected for this work.

Figure 4 illustrates the resolution and distribution of mesh \#2, which is generated using ANSYS® ICEM containing only hexahedral elements.

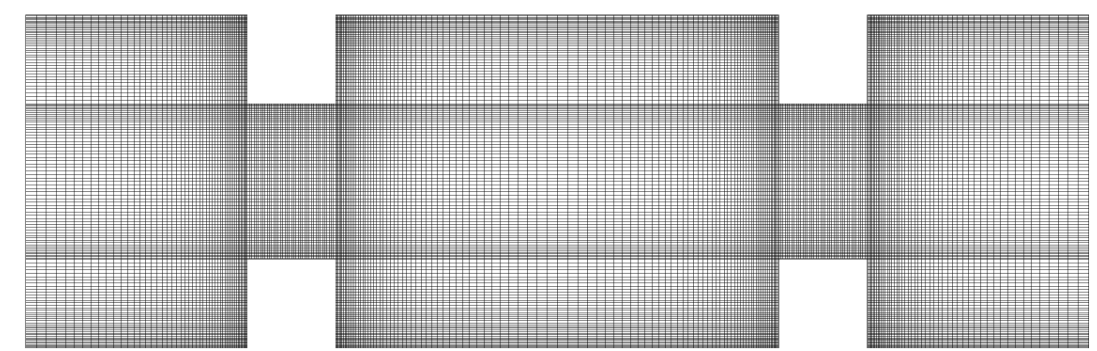


Figure 4. Finite volume chosen mesh (\#2): 17k nodes per baffled cell

Table 2. Mesh sensitivity analysis results $\left(R^{2}\right)$ where results from mesh \#1 are used as the based for comparison

\begin{tabular}{|c|c|c|c|c|c|}
\hline \multicolumn{6}{|c|}{ Line 1} \\
\hline Mesh \# & \# Nodes ${ }^{*}$ & Stroke 1 & Stroke 2 & Stroke 3 & Stroke 4 \\
\hline 1 & $38 \mathrm{k}$ & - & - & - & - \\
\hline 2 & $17 \mathrm{k}$ & 0.999 & 0.997 & 0.999 & 0.945 \\
\hline 3 & $10 \mathrm{k}$ & 0.999 & 0.997 & 0.997 & 0.896 \\
\hline 4 & $6 \mathrm{k}$ & 0.994 & 0.989 & $(0.999)^{\prime}$ & 0.948 \\
\hline 5 & $3 \mathrm{k}$ & 0.982 & 0.952 & 0.993 & 0.953 \\
\hline \multicolumn{6}{|c|}{ Line 2} \\
\hline Mesh \# & \# Nodes ${ }^{*}$ & Stroke 1 & Stroke 2 & Stroke 3 & Stroke 4 \\
\hline 1 & $38 \mathrm{k}$ & - & & - & - \\
\hline 2 & $17 \mathrm{k}$ & 0.988 & 0.988 & 0.966 & 0.972 \\
\hline 3 & $10 \mathrm{k}$ & 0.986 & 0.994 & 0.948 & 0.933 \\
\hline 4 & $6 \mathrm{k}$ & 0.968 & 0.925 & 0.893 & 0.867 \\
\hline 5 & $3 \mathrm{k}$ & 71 & 0.760 & 0.918 & 0.621 \\
\hline \multicolumn{6}{|c|}{ Line 3} \\
\hline Mesh \# & \# Node & Stroke 1 & Stroke 2 & Stroke 3 & Stroke 4 \\
\hline 1 & $38 \mathrm{k}$ & - & - & - & - \\
\hline 2 & 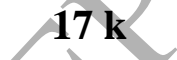 & 0.997 & 0.983 & 0.994 & 0.983 \\
\hline 3 & $10 \mathrm{k}$ & 0.996 & 0.989 & 0.989 & 0.962 \\
\hline 4 & $6 \mathrm{k}$ & 0.984 & 0.978 & 0.975 & 0.965 \\
\hline 5 & $3 \mathrm{k}$ & 0.924 & 0.952 & 0.911 & 0.922 \\
\hline
\end{tabular}

"The values of number of nodes are per baffled cell.

\section{Results and discussions}

\subsection{Profile development}

Previous CFD work on oscillatory baffled reactors by Jian (Jian, 2002) showed that flow patterns in an OBR become repeatable and achieved a quasi-steady-state after 5-7 oscillation 
cycles. Following the same methodology, the volume-weighted averaged strain rate as a function of time is shown in Figure 5, displaying a similar quasi-steady state after cycle 4 . In this work, cycle-averaged parameters generated between cycles 6 to 10 are used for comparison.

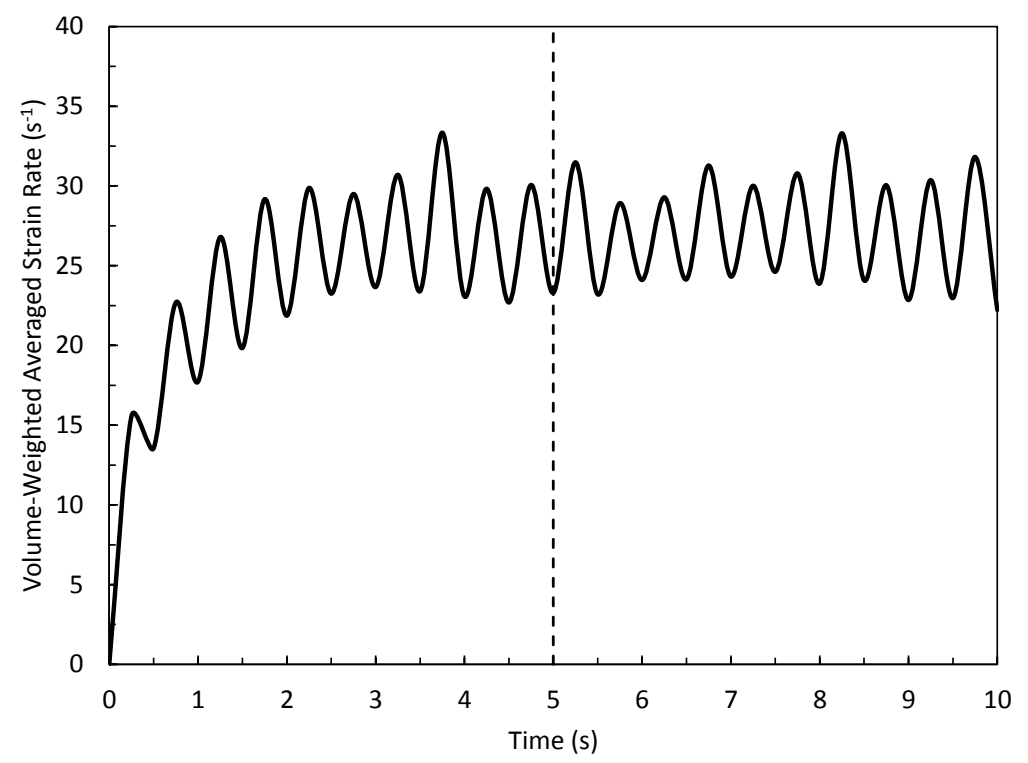

Figure 5. Convergence of strain rate with time in Fluent

\subsection{Sensitivity test of SPH particles}

Figure 6 shows the cycle-averaged velocity magnitude profiles of Line 3 (see Figure 3), generated by SPH, at strokes 1 and 3 (see Figure 1) for three different resolutions in terms of the initial particle distribution spacing, $\Delta x$. Strokes 1 and 3 were chosen because the flow at these instances experiences the maximum local velocities, leading to potential lower rates of convergence. A clear convergence in the cycle-averaged velocities is observed with decreasing $\Delta x$, and the results become resolution independent at $\Delta x=0.00025 \mathrm{~m}$, which is therefore chosen for the investigation. 

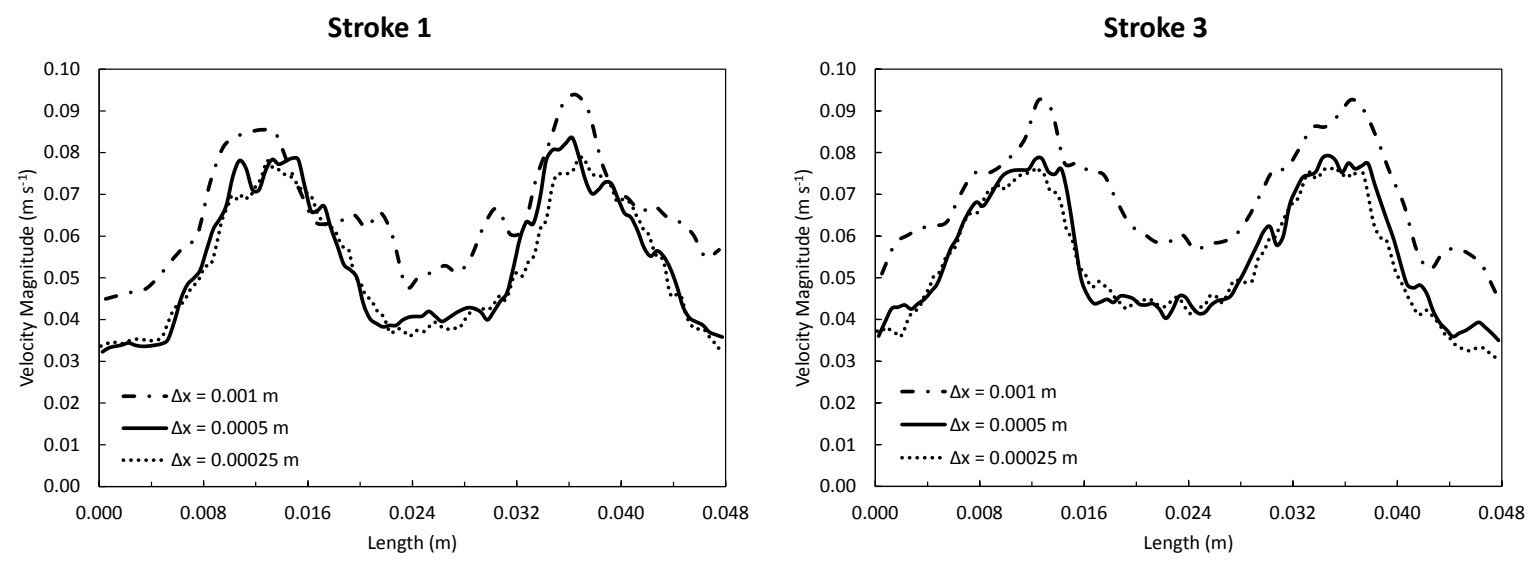

Figure 6. SPH cycle-averaged velocity magnitude profiles of Line 3 for different $\Delta x$ values

\subsection{Flow patterns and velocity profiles}

\subsubsection{Flow patterns}

Figure 7 shows the cycle-averaged flow patterns modelled by both the FV and the SPH with an additional density-smoothing function (SPH D-S for short) at strokes 1 to 4 of an oscillation cycle (see Figure 1). Both methods predict similar eddy formation throughout the oscillation, leading to good comparison. The subtle differences between the two models are the relative size and the intensity (by color) of eddies. The intensity of flow restriction in strokes 1 and 3 is higher for SPH D-S than that for FV, resulting in larger recirculating velocities at the top and bottom of the baffled cell, causing the eddy structures generated during strokes 2 and 4 to remain closer to the baffle's walls. On the contrary, results generated by the FV have slightly larger velocities along the center of the baffled domain, enhancing a small displacement of eddies towards the center of the baffled cell. This slight difference in eddy displacement is reflected in the velocity profiles presented in the next subsection and the maximum velocities listed in Table 3. Flow patterns observed during strokes 1 and 2 and eddy structures formed in strokes 2 and 4 are of high resemblance for the two 
methodologies nonetheless. Overall, the results show that the SPH D-S is a viable method in modelling flows in OBR.
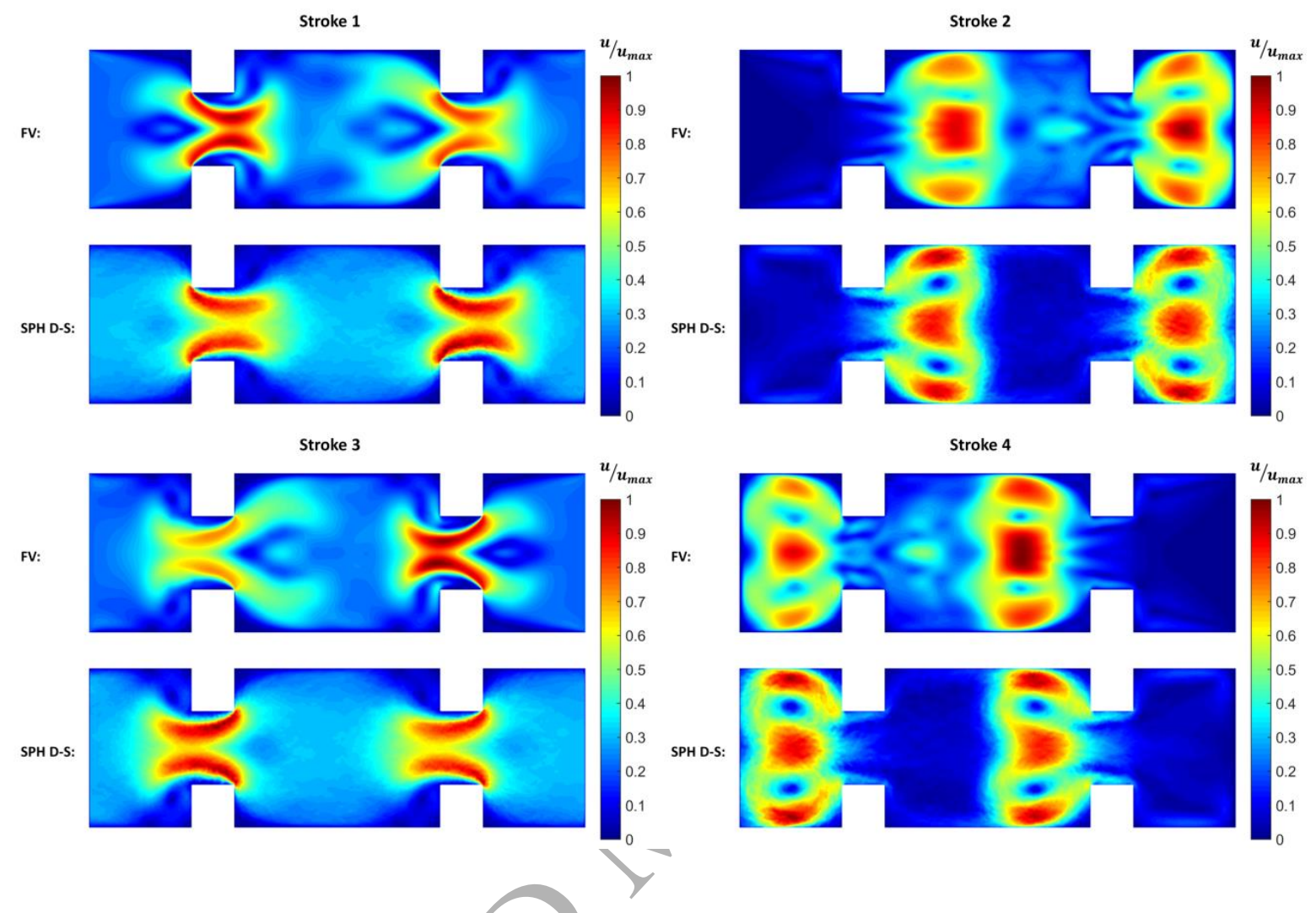

Figure 7. Cycle-averaged velocity magnitude contours at strokes 1 to 4 for FV and SPH D-S

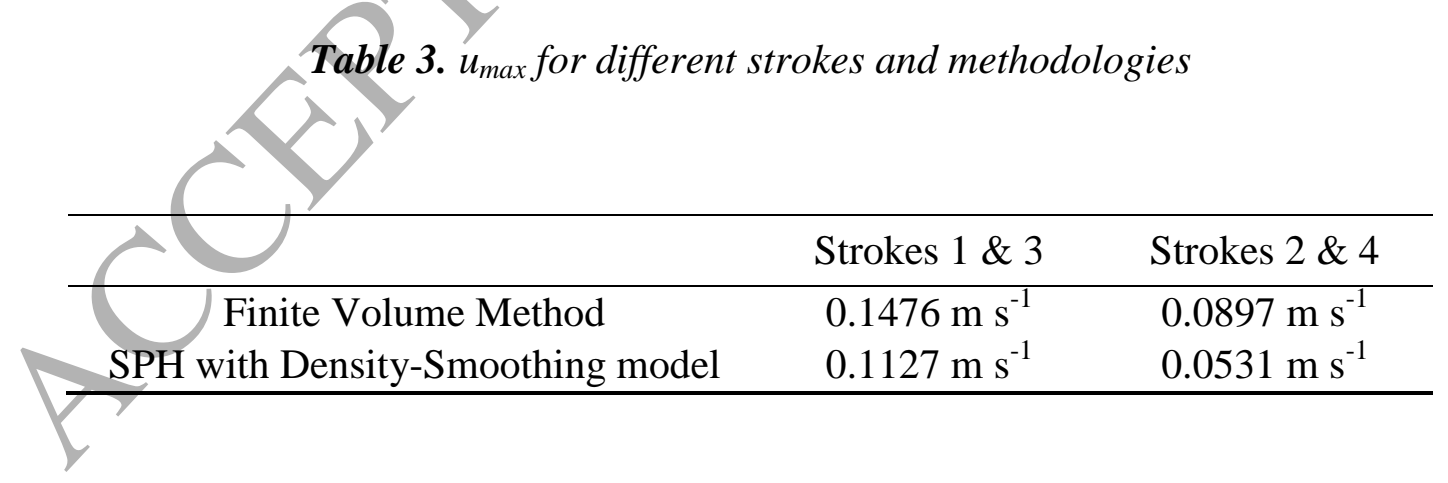

\subsubsection{Velocity profiles}

The cycle-averaged velocity magnitude profiles at Lines 1, 2 and 3 computed by the FV, the standard SPH and SPH D-S methodologies are shown in Figures 8 - 10, respectively; the 
density-smoothing function was added in a separate simulation to assess its effect individually.

Figure 7 neatly displays how two noticeable high velocity regions (red color) occur through the baffle constriction during strokes 1 and 3, which is shown in Figure 8 as a double-peak velocity profile across Line 1 . The velocities obtained with the SPH methodology alone do not display the expected double-peak pattern as the FV did in the forward (1) and reverse (3) strokes, but exhibit parabolic-like characteristics which indicate excessive localized density fluctuations due to the weakly compressible limitation across the constriction of the baffle. This effect is then minimized when the density-smoothing (D-S) function is introduced to limit the "noise" in density distribution. In doing so, the double-peak velocity profiles are reproduced during strokes 1 and 3, highly resembling those modelled by the FV method. Figure 7 also provides qualitative evidence of how, during strokes 2 and 4, the changes in direction of the flow lead to local stalling across the baffle constriction, which translates into lower and more uniform velocity components in the baffle constriction area as shown in Figure 8. The results from the SPH simulation echo the above nonetheless; again the results from the SPH D-S improve the accuracy of the velocity field and are similar to those provided by the FV method.

For the given geometry and operating conditions, symmetrical-mirrored flow patterns and similar velocity magnitudes between the peak and trough are expected (see Figure 7). The velocity profiles predicted by SPH D-S (in Figure 8) at strokes 1 and 3 are indeed very similar in both shape and magnitude with a maximum difference of $4.7 \%$, while the results computed by the FV approach at the same strokes have a larger discrepancy, of $18.5 \%$. A similar outcome is likewise seen for strokes 2 and 4. 
Stroke 1

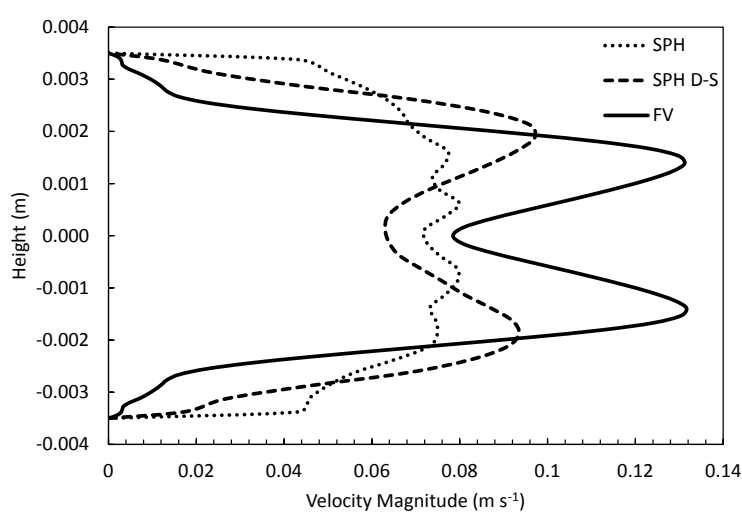

Stroke 3

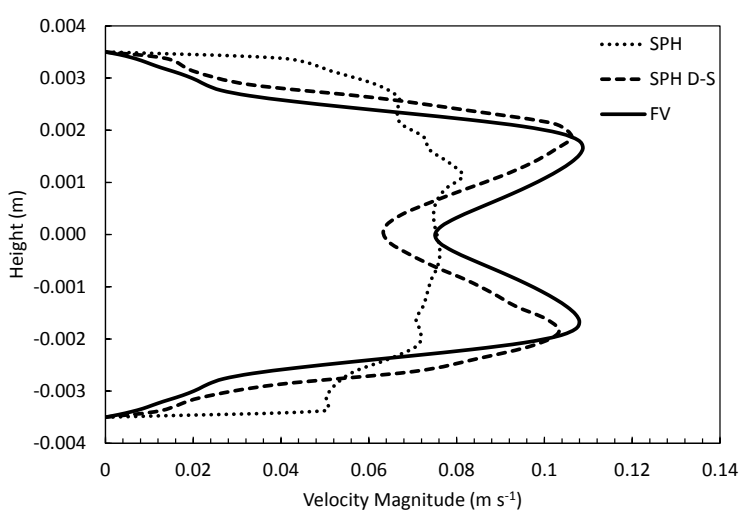

Stroke 2

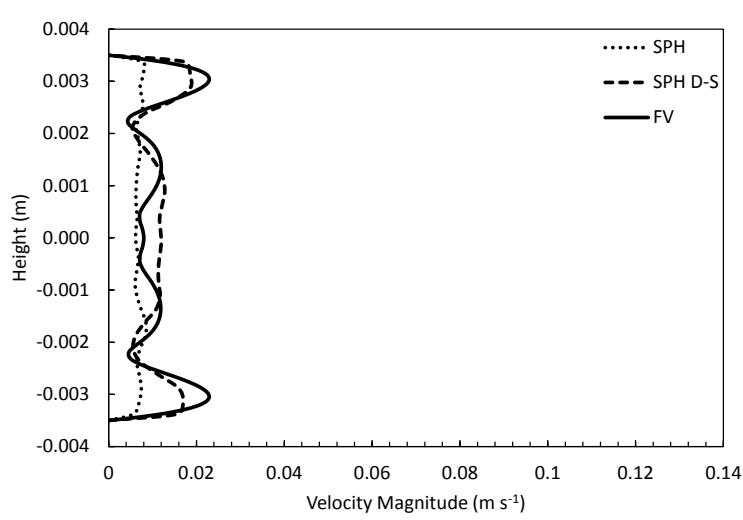

Stroke 4

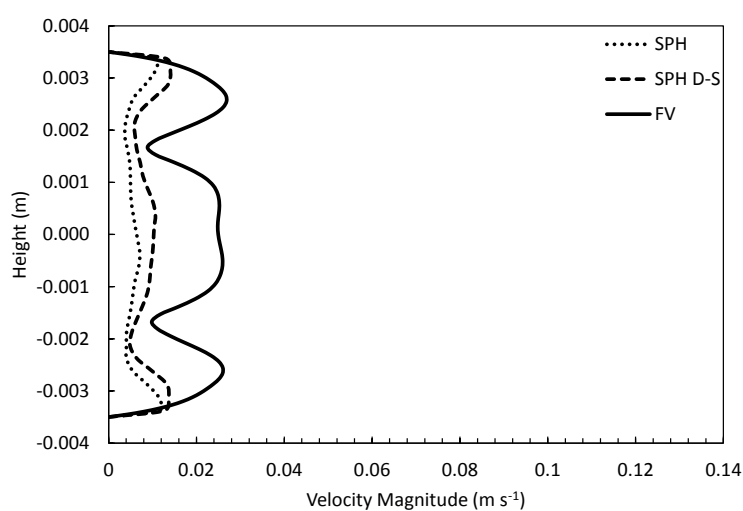

Figure 8. Cycle-averaged velocity magnitude profiles of Line 1 for SPH, SPH D-S and FV methods

The cycle-averaged velocity magnitude profiles across Line 2 (in the middle of the baffled cell, see Figure 3) are shown in Figure 9 for the FV, SPH and SPH D-S approaches. It is seen that the velocity profiles at the strokes 1 and 3 by the FV not only differ in velocity amplitude with a maximum error of $8.7 \%$, but also differ in shape. Conversely, the velocity profiles by the SPH D-S method display repeatable cyclic flow with a maximum error of $0.5 \%$ at the same strokes. It demonstrates again that a density-smoothing method is essential to provide regular and predictable flow patterns with $\mathrm{SPH}$, showing good reproducibility and consistency for modelling oscillatory transient problems. 
For strokes 2 and 4 representing the end of both the forward and backward strokes respectively, the comparisons of velocity profiles are better for both the FV and SPH D-S methods; however the magnitudes for the former are larger than for the latter.

These differences in the magnitude and shape of velocity profiles among FV strokes, and between FV and SPH D-S, at Line 2, are due to the earlier mentioned phenomenon: Figure 7 showed that while eddy structures generated by SPH D-S remain close to the walls of the baffles, eddies observed in FV solutions are slightly displaced towards the center of the baffled cell. This leads to an increment in velocity magnitude at Line 2, in comparison with those obtained with SPH D-S. This eddy displacement shown in FV results occurs at every cycle; the distance travelled by the eddies remains significantly constant throughout cycles, leading to a quasi-steady-state, hence a stable cycle-averaged velocity field is obtained (Figure 7). However, subtle alterations in the distances travelled by eddies from cycle to cycle manifest into significant differences in the magnitude of velocity and the shape of velocity profiles at Line 2 among FV strokes. This is clearly observed in Figure 11, where velocity magnitude profiles, obtained with FV and extracted at Line 2, are presented at strokes 1 to 4 for different oscillatory cycles. Likewise, Figure 12 shows the analogous for SPH D-S. Undoubtedly, SPH D-S does a better job at producing cycle-repeatable results than $\mathrm{FV}$, especially for velocity profiles at a vertical centered line (at the middle of the baffled cell). Nonetheless, despite this phenomenon and its impact on velocity profiles at Line 2, both methodologies present very similar cycle-repeatable velocity fields across the entire domain, capturing almost identical eddy generation patterns, as stated in section 4.3.1. 

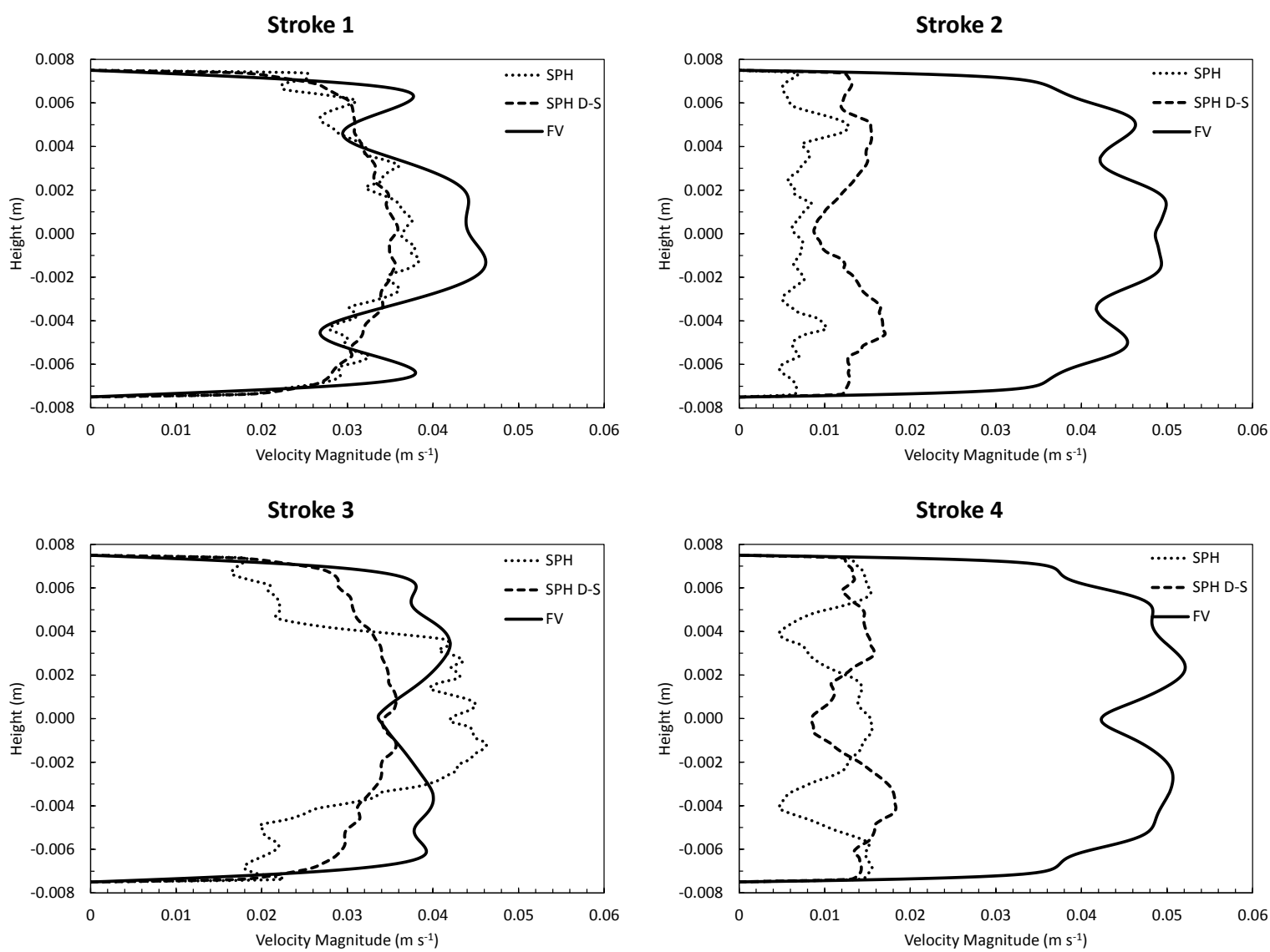

Figure 9. Cycle-averaged velocity magnitude profiles of Line 2 for SPH, SPH D-S and FV

\section{methods}

The cycle-averaged velocity magnitude profiles shown in Figure 10 are measured across a horizontal center line (Line 3 in Figure 3). Again, the standard SPH approach suffers from noise arising from its density distribution, consequently, the velocity magnitudes for all strokes display small fluctuations that have a dampening effect on the overall flow profile, in particular in strokes 2 and 4 where the velocity components are small. These density fluctuations and the associated velocity dampening effect are eliminated by the use of SPH D-S. As earlier mentioned, the numerical solution for strokes 1 and 3, and strokes 2 and 4, should ideally yield a profile that mirrors one another; this is especially expected at Line 3, as it is positioned across the axial direction. Both FV and SPH D-S methodologies successfully 
predict mirror-shaped velocity profiles among strokes, and show good agreement and resemblance among one another. Local differences in velocity magnitude are the results of the aforementioned eddy displacement produced by FV.
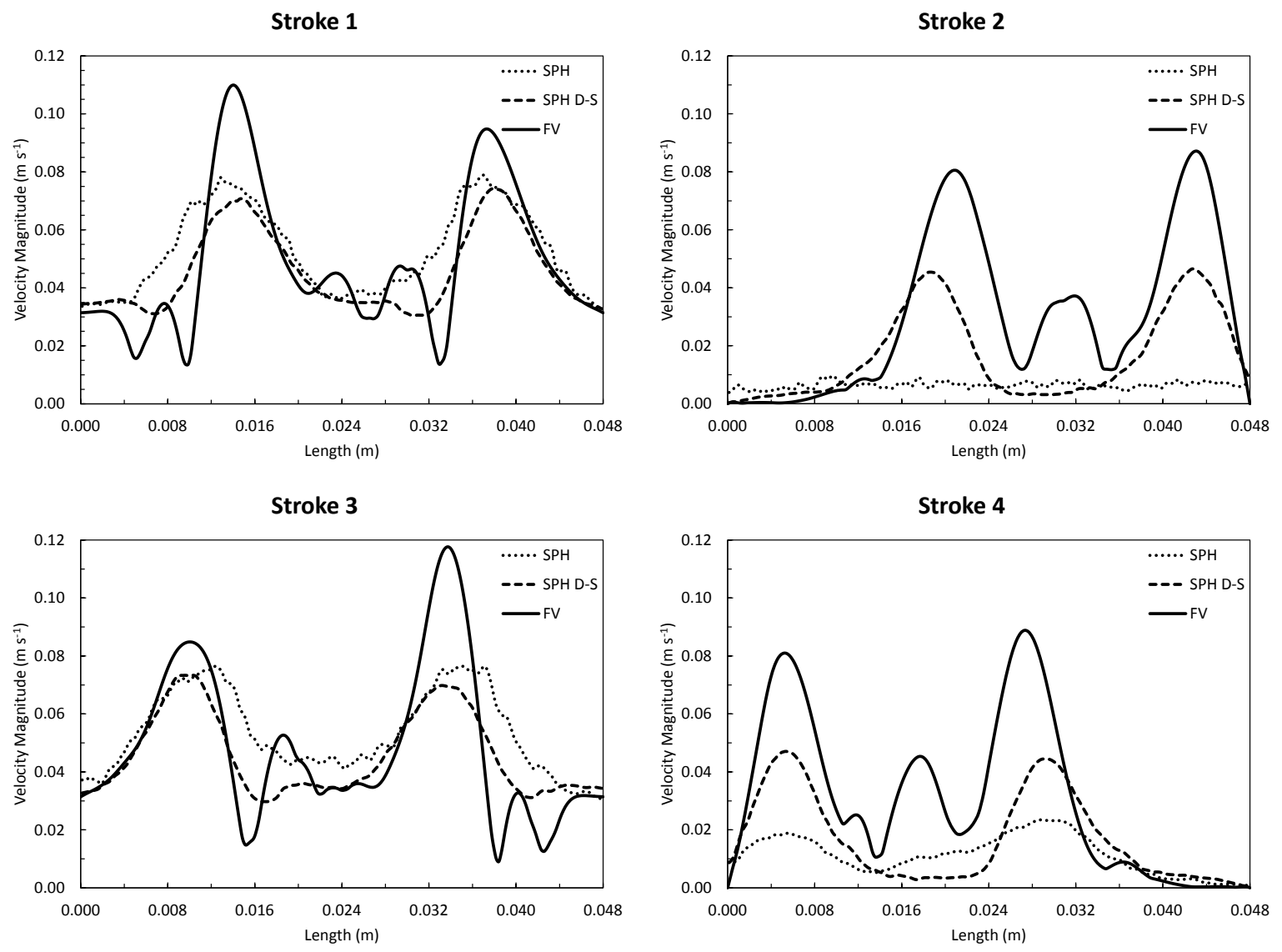

Figure 10. Cycle-averaged velocity magnitude profiles of Line 3 for SPH, SPH D-S and FV methods 

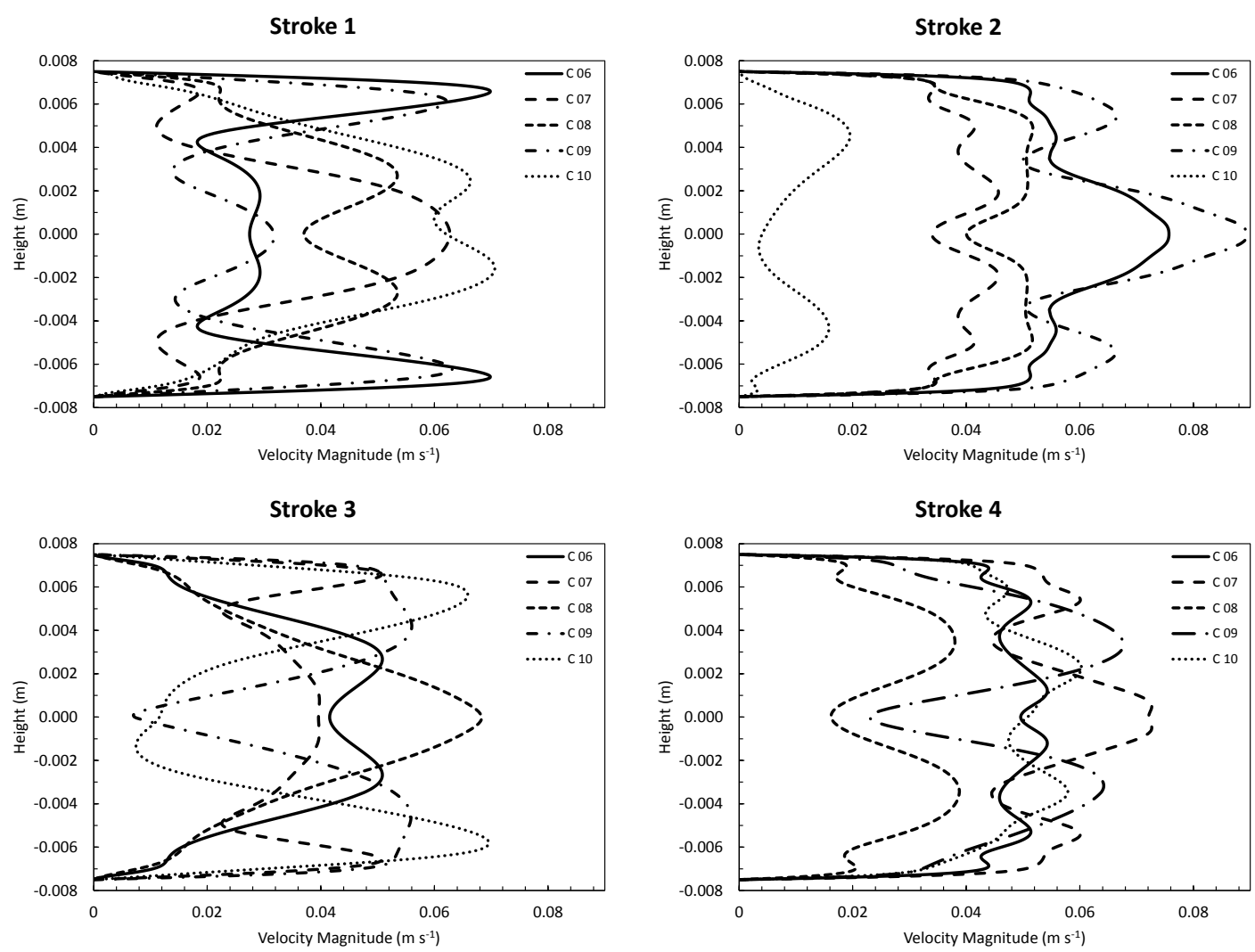

Figure 11. Velocity magnitude profiles of Line 2 obtained with Finite Volume (FV) method for different oscillatory cycles 

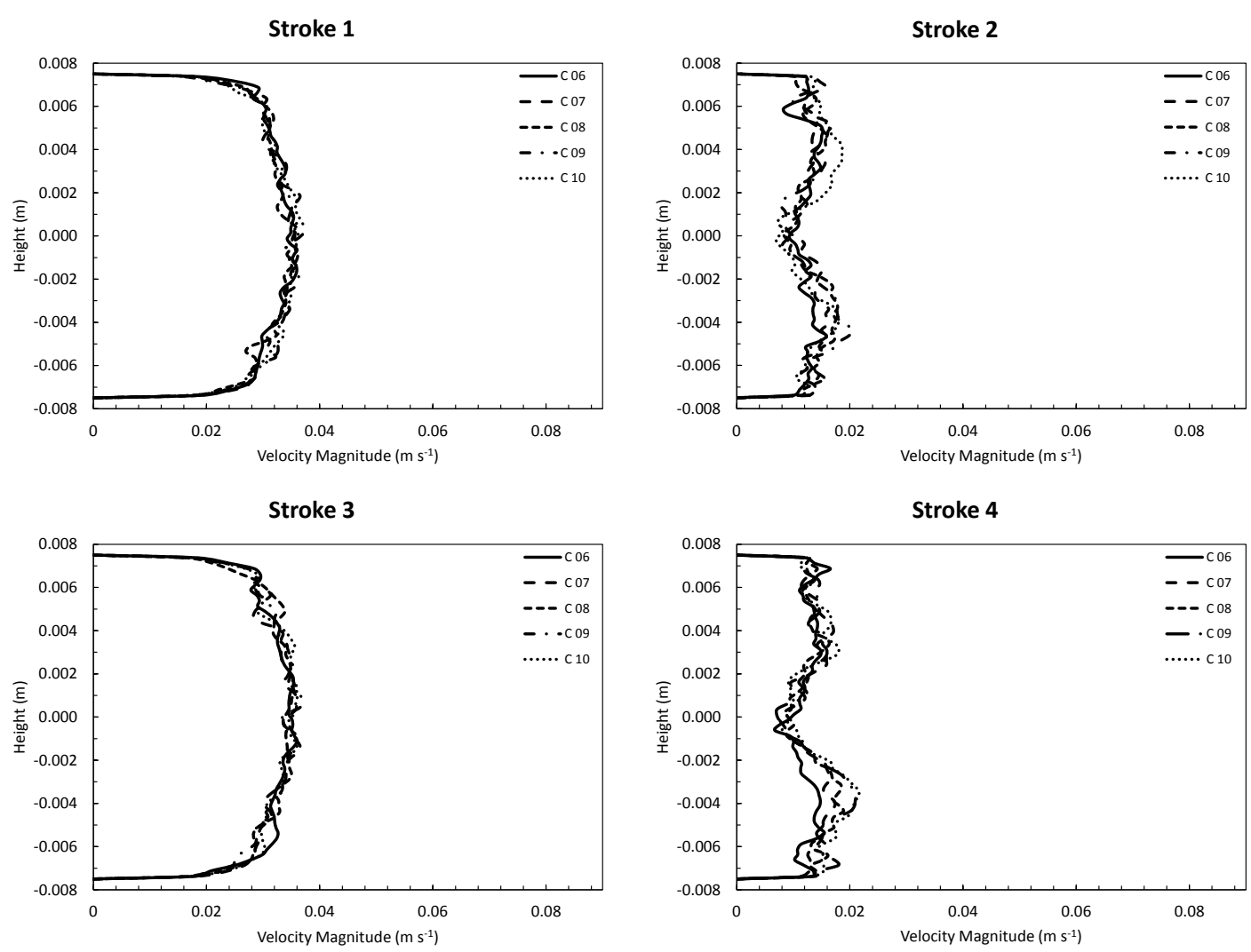

Figure 12. Velocity magnitude profiles of Line 2 obtained with SPH Density-Smoothing (SPH D-S) method for different oscillatory cycles

\subsection{Mixing assessment}

Diverse methods and indexes have been used in the past in order to quantitatively assess mixing efficiency in OBR, for example, the velocity ratio (Fitch et al., 2005; Jian \& Ni, 2005; Manninen et al., 2013) and stretch rates (Mackley \& Neves Saraiva, 1999; Roberts \& Mackley, 1995). Simulated data obtained with the SPH can directly be utilized to do the same analysis.

\subsubsection{Velocity ratio}

The previously used axial to radial velocity ratio $\left(R_{V}\right)$ is defined as: 


$$
R_{V}(t)=\frac{\sum_{i=1}^{N_{T}}\left(\left|u_{x, i}\right| \frac{m_{i}}{\rho_{i}}\right)}{\sum_{i=1}^{N_{T}}\left(\left|u_{\mathrm{y}, i}\right| \frac{m_{i}}{\rho_{i}}\right)}
$$

where $u_{x, i}$ and $u_{y, i}$ are, respectively, the axial and radial velocity components of a particle $i$, and $N_{T}$ is the total number of fluid particles in the domain. By directly utilizing the velocity data generated by the SPH D-S method, the cycle-averaged velocity ratio is obtained and plotted against the period of an oscillatory cycle in Figure 13; the shape of which is the same as that reported by Jian and Ni (Jian \& Ni, 2005). Past work (Fitch et al., 2005; Jian \& Ni, 2005; Manninen et al., 2013) correlated $R_{V}$ as a function of oscillatory Reynolds number $\left(R e_{o}\right)$ for fluids of different viscosities in OBRs, where an inversely proportional relationship was established for $\mathrm{Re}_{\mathrm{o}}<1000$. The averaged $R_{V}$ value of 3.18 in this work is slightly higher than the aforementioned work (Jian \& Ni, 2005), due to the lower oscillatory Reynolds number $\left(R e_{o}=471\right)$ under which the current study was performed.

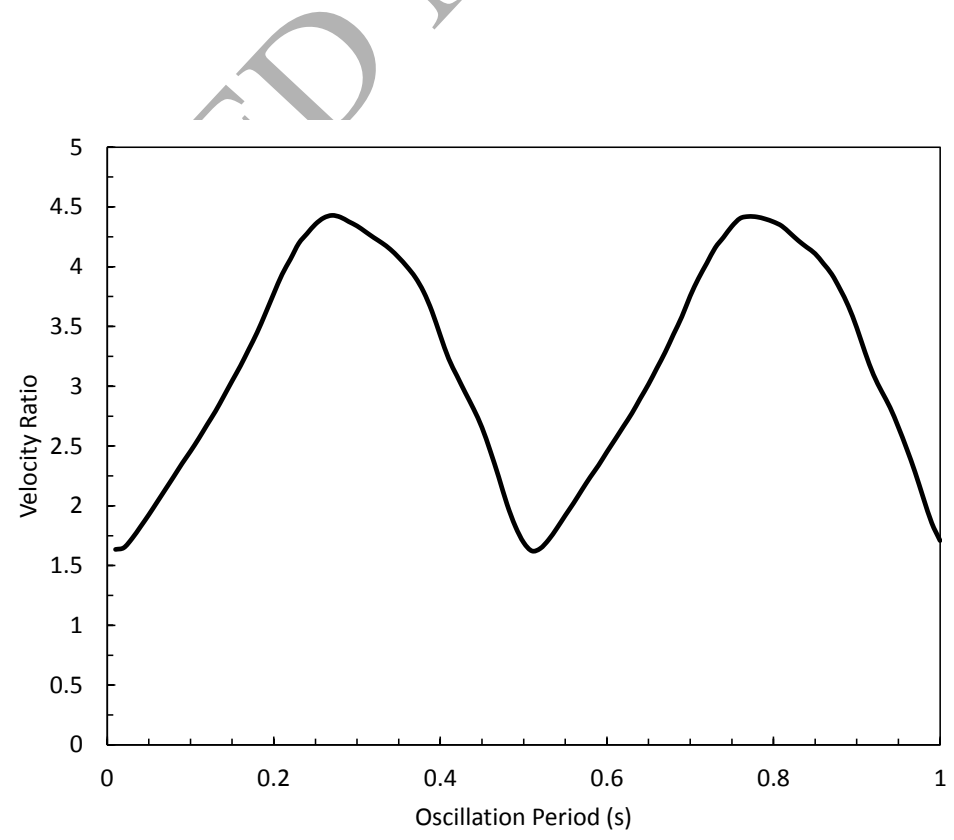

Figure 13. Cycle-averaged velocity ratio for the period of an oscillatory cycle 


\subsubsection{Stretch rates}

Another way of quantifying the rates of mixing in a system, due to its fluid dynamics, is by analysing the rates at which infinitesimal lines of the fluid domain are stretched (Ottino, 1989). This appraoch requires Lagrangian tracking of these infintesimal lines that compose the domain under evaluation. Advantageously, by modelling the flow field with SPH, the necessity of superimpossing a Largrangian tracer whose movement is integrated based on an Eulerian velocity field (Mackley \& Neves Saraiva, 1999; Roberts \& Mackley, 1995) is avoided. It is an easy task to assign an infinitesimal line to each fluid particle that comprises the domain in SPH; these lines are then ascribed an initial orientation (at time $=0 \mathrm{~s}$ ) relative to the axis in the $x$-direction, defined by the anticlokwise angle $\theta$. The rate of rotation is thus given by:

$$
\frac{d \theta}{d t}=\cos ^{2} \theta \frac{\partial u_{y}}{\partial x}+\sin \theta \cos \theta\left(\frac{\partial u_{y}}{\partial y}-\frac{\partial u_{x}}{\partial x}\right)-\sin ^{2} \theta \frac{\partial u_{x}}{\partial y}
$$

and the instantaneous exponential stretch rate of an infitesimal line is defined as:

$$
\frac{d(\ln l)}{d t}=\sin ^{2} \theta \frac{\partial u_{y}}{\partial y}+\sin \theta \cos \theta\left(\frac{\partial u_{y}}{\partial x}+\frac{\partial u_{x}}{\partial y}\right)+\cos ^{2} \theta \frac{\partial u_{x}}{\partial x}
$$

The time-averaged exponentatial stretch rate $S(t)$ of a line can be integrated as:

$$
S(t)=\frac{1}{t} \int_{0}^{t} \frac{d(\ln l)}{d t} d t
$$

The above is then volume-averaged for all the lines comprising the system $-\bar{S}(t)-$ and plotted with time, giving an asymptotic value $\bar{S}_{a s}$. Exponential stretch rates have units of s ${ }^{-1}$, 
and are scaled with the frequency of the oscillation $(f)$ in order to obtain dimensionless values. Roberts and Mackley (Roberts \& Mackley, 1995) obtained $\bar{S}(t)-\operatorname{Re}$ and $\bar{S}(t)-S t$ relations, while the effect of $R e_{o}$ on $\bar{S}(t)$ was later established by Mackley and Neves Saraiva (Mackley \& Neves Saraiva, 1999). Based on their findings, $\bar{S}_{a s}$ values between 0.5 and 1 are expected for the operational conditions of the current system under evaluation; the asymptotic values in Table 4 fall within the range. Figure 14 shows the evolution of $\bar{S}(t)$ for different initial values of $\theta$; the profiles and trends are identical to those of the aforementioned research works.

Table 4. Effect of the initial orientation of the infinitesimal lines on the asymptotic value of the systems exponential stretch rate

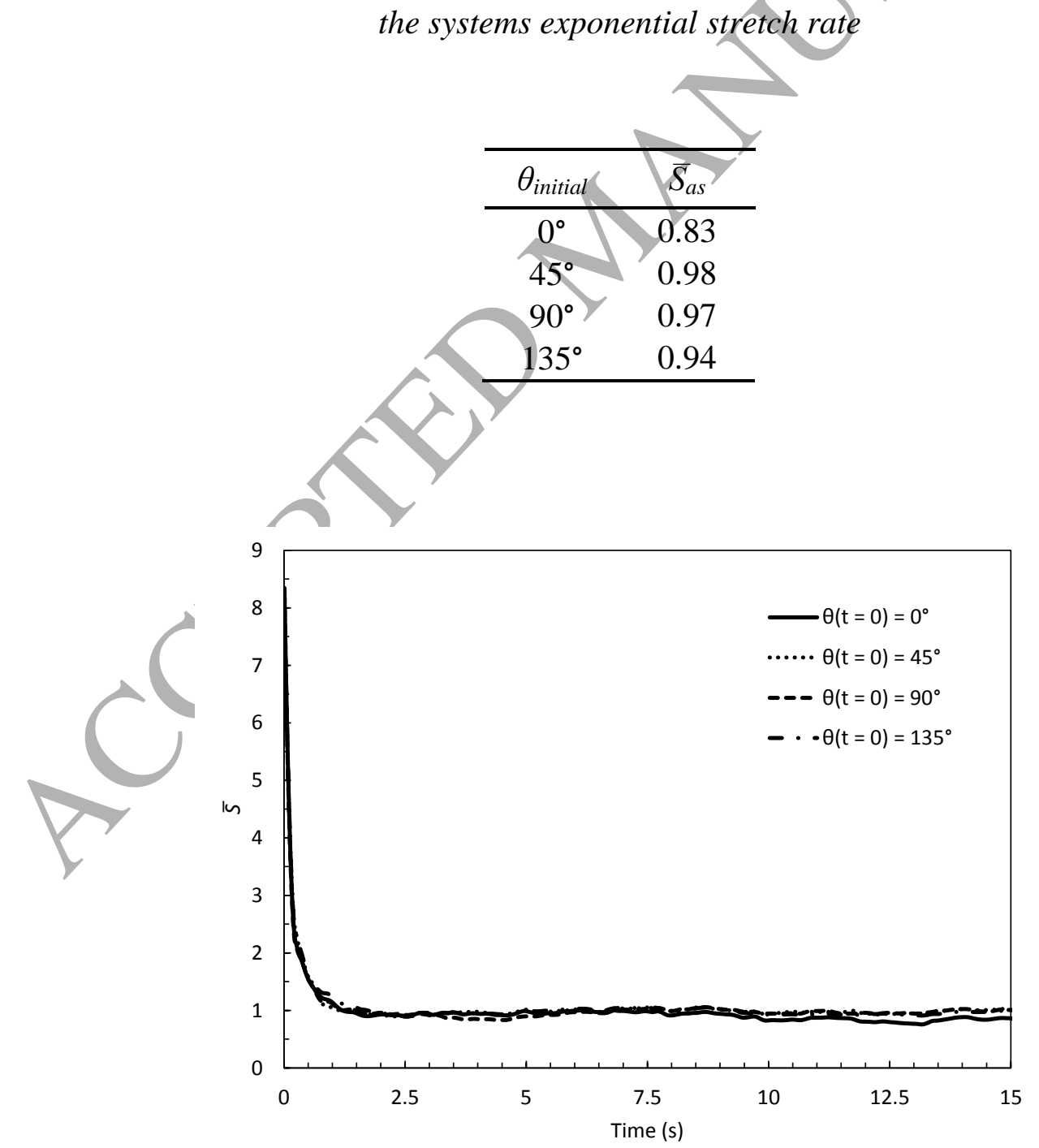


Figure 14. Effect of the initial orientation of the infinitesimal lines on the time evolution of the time-averaged exponentatial stretch rate

\subsubsection{Distribution of neighboring fluid particles}

Using the SPH approach, an alternative mixing assessment can be proposed by defining the domain as two identical immiscible fluids; these fluids can initially be displayed in serial or in parallel, as shown in Figures 15 and $16($ time $=0 \mathrm{~s})$. As the simulation advances with time, visual and qualitative assessment of axial and radial mixing efficiency are observed in Figures 15 and 16, respectively.

Time $=0 \mathrm{~s}$

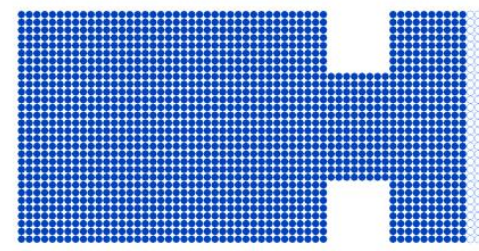

Time $=25 \mathrm{~s}$

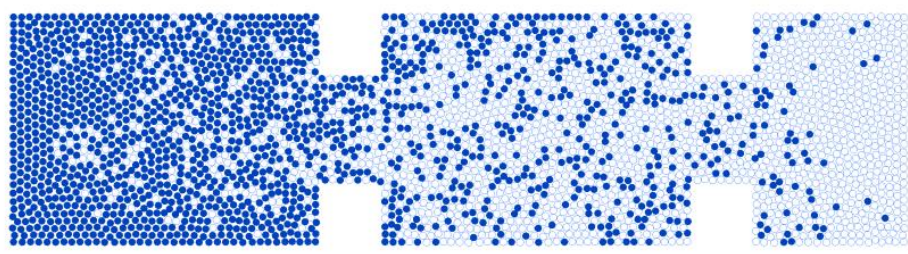

Time $=\mathbf{5 0} \mathrm{s}$

- Fluid A

Fluid B

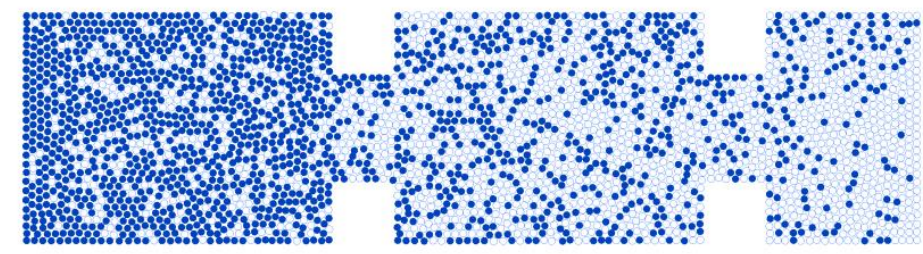

Figure 15. Qualitative axial mixing assessment for $S P H D-S$ and $\triangle x=0.0005 m$ 


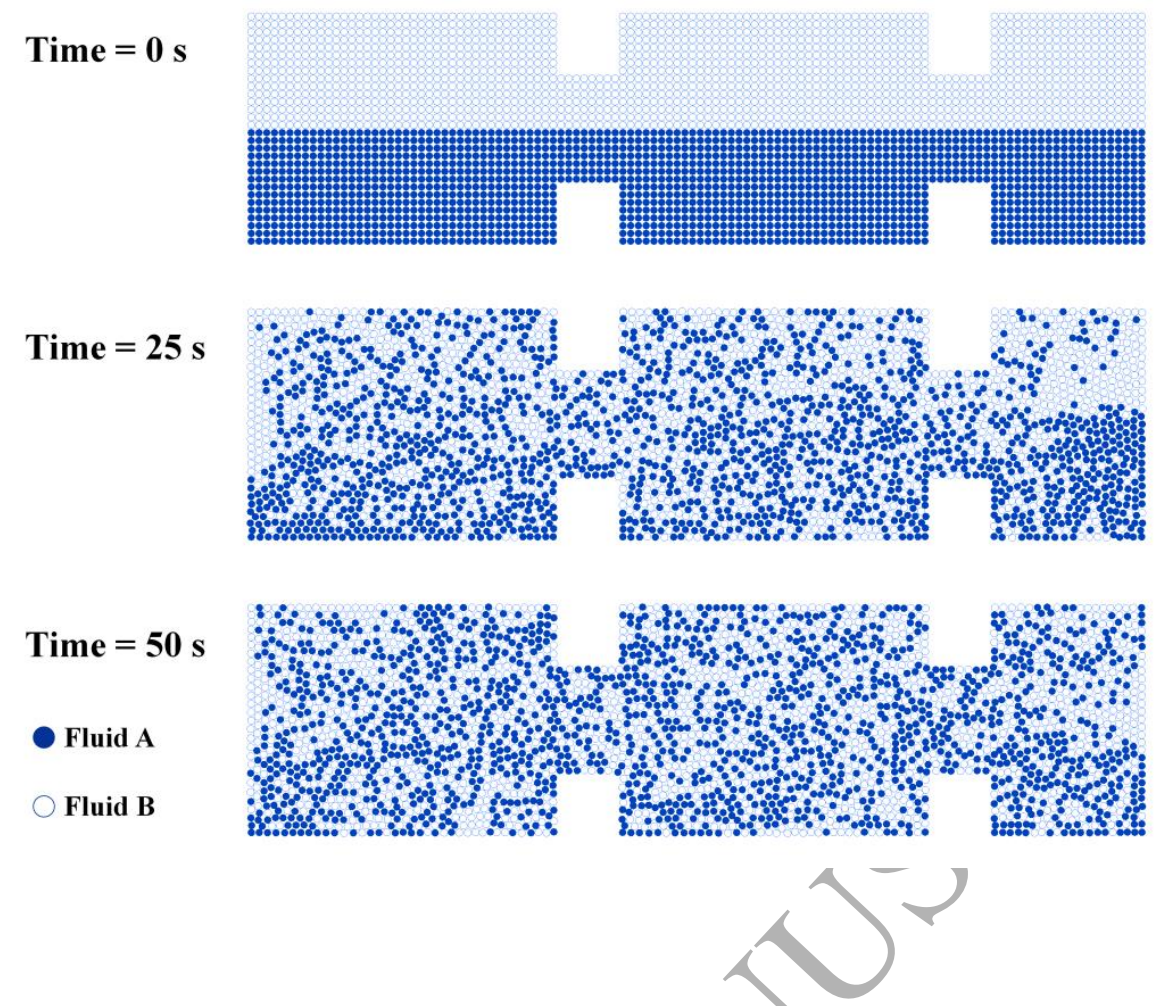

Figure 16. Qualitative radial mixing assessment for $S P H D-S$ and $\triangle x=0.0005 m$

The evolution of mixing efficiency with time can be obtained by quantifying, for each particle, how many of its neighboring particles are of Fluid A, and how many of its neighbors are of fluid B. Numerically, this is done as follows. Let each particle $i$ carry a dimensionless binary variable $J_{i}$, whose value can be 0 (if it is a Fluid A particle) or 1 (Fluid B). By doing so, a time-dependent dimensionless "neighboring mixing index", $N M(t)$, is defined for each particle as:

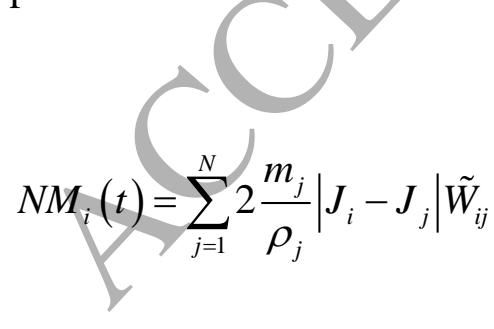

where the kernel $W_{i j}$ has been corrected using the Shepard filter (Panizzo, 2004), in order to avoid irregularities with particles close to the boundaries, as those will have a lower number of neighboring fluid particles than particles in the bulk. $\tilde{W}_{i j}$ is defined as: 


$$
\tilde{W}_{i j}=\frac{W_{i j}}{\sum_{j=1}^{N} \frac{m_{j}}{\rho_{j}} W_{i j}}
$$

The neighboring mixing index is averaged over all the particles comprissing the fluid domain. At time zero, every Fluid A particle has a certain number of neighboring particles, all of which are of Fluid A; likewise, every Fluid B particle only has Fluid B neighbors. This will be true for all particles, except those at the interface that separates the two types of fluids, hence yielding a $N M(t=0 s) \approx 0$. On the contrary, a fully mixed system is achieved when each particle has an equal number of neighbors of Fluid A and Fluid B, resulting in $N M=1$.
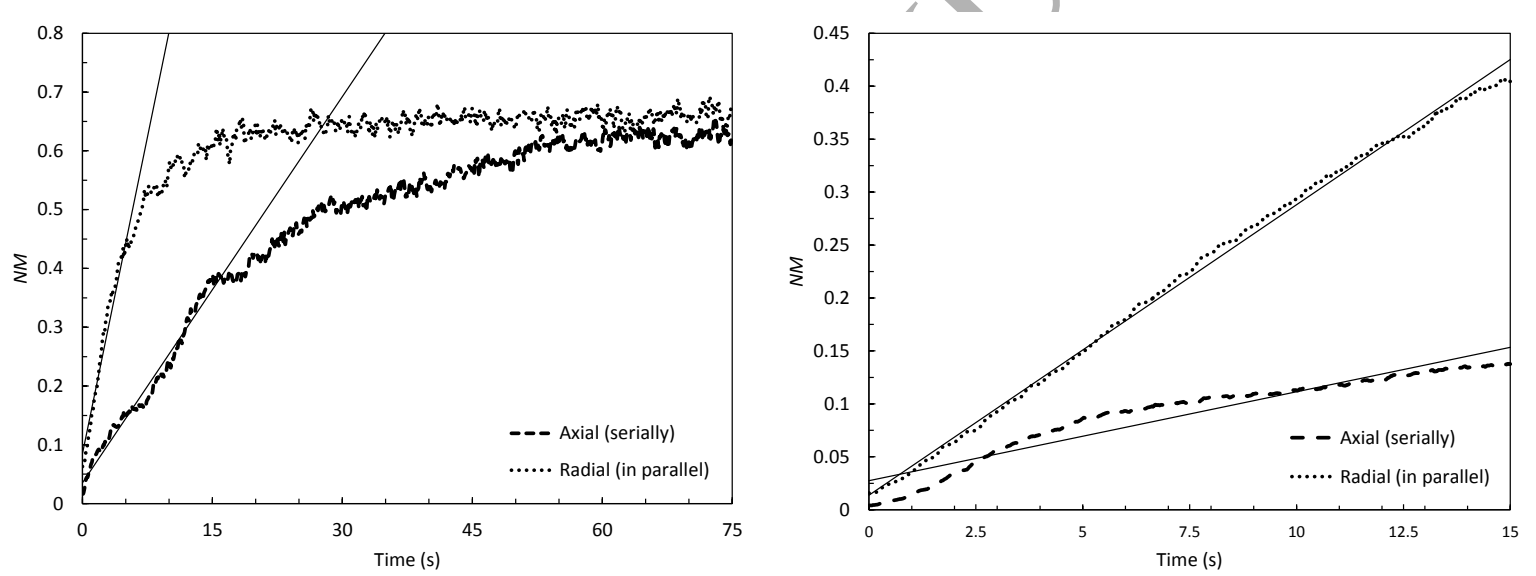

Figure 17. Effect of the initial display of fluids $A$ and $B$ on the time evolution of $N M$ for $\Delta x=$ $0.001 m$ (left) and $\Delta x=0.00025 m($ right $)$

Figure 17 plots the $N M$ as a function of time for both fluid placements as shown in Figures 15 and 16, for two initial particle distribution spacing $(\Delta x)$. It is seen that both the axial and radial neighboring mixing indices increase with time and level off at about 0.6 (Figure 17 left). This is expected, as there is no interaction between eddies at the selected operational conditions. Using a smaller $\Delta x$ (higher resolution), increments of both axial and radial 
neighboring mixing indices with time are slower, as the domain is composed by a larger number of particles, taking longer times for them to intermix. Figure 17 also shows a better mixing performance in the radial than in the axial direction as the asymptotic value is reached faster when fluids A and B are initially displayed in parallel. Using the rate at which $N M$ changes with time (i.e. $d M N / d t$ ) during the first oscillatory cycles for both initial fluid placements, a new index for plug flow $(P F)$ can be defined as:

$$
P F=\frac{(d N M / d t)_{\text {axial }}}{(d N M / d t)_{\text {radial }}}
$$

The lower the value of $P F$, the better the plug flow behavior the system can achieve. The slopes in Figure 17 together with $P F$ values are listed in Table 5 where a small $P F$ value (0.31) is consistently obtained regardless of $\Delta x$, indicating near plug flow performance. Note that computational time constrictions prevented simulaitions with small $\Delta x$ values from running long enough for an asymptotic $N M(t)$ value to be achieved. However, for the sake of demonstration, the results for $\Delta x=0.001 \mathrm{~m}$ are presented here, which accurately predict the ratio of axial to radial $N M$ change with time.

Table 5. Change rates of $N M$ and PF values for different flow field resolutions $(\triangle x)$

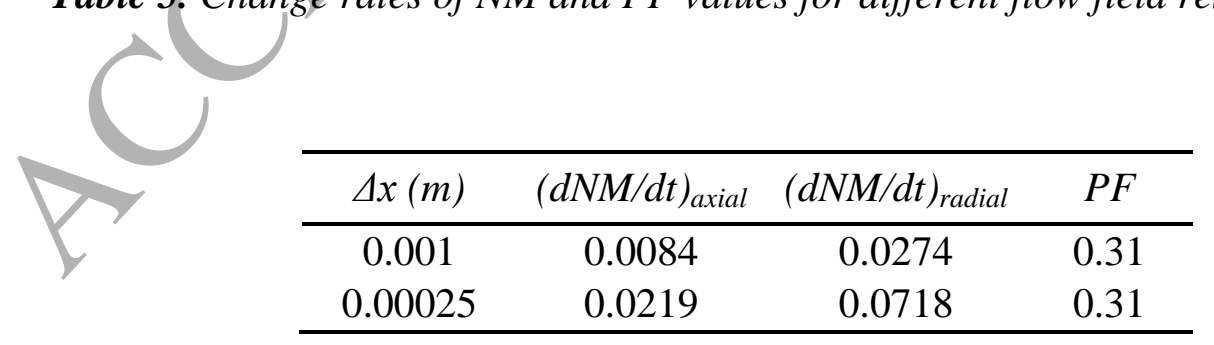


It should be noted that a more complex and computationally expensive EulerianLagrangian coupled solver (Discrete Phase Model) is required by the FV methodology to provide similar information of individual particles as the one obtained with the SPH.

\section{Conclusions}

In this paper, SPH, a relatively new Lagrangian approach, has successfully been implemented and utilized for the first time to model and predict symmetrical flow patterns and to assess mixing efficiency in a 2-dimensional OBR system.

The SPH has effectively captured the expected flow characteristics in an oscillatory baffled reactor and produced clear higher velocity regions at the baffle constriction during strokes 1 and 3, and eddy formation during the change in direction at strokes 2 and 4 . The density-smoothing function in SPH is important to offset density fluctuations stemmed from the weakly compressible model. The results from SPH D-S provide a more consistent quasisteady-state flow and show a higher degree of cycle-repeatability than that from its Eulerian counterpart.

An added advantage of SPH is that it allows quantitative assessments of mixing without the need for additional models like Eulerian based methods, due to its readily available information of individual fluid particles. This work has not only demonstrated its potential to easily implement the existing methods to quantify mixing, such as velocity ratio and stretch rates, but also proposed new indexes for assessing mixing and plug flow efficiency by making full use of SPH's capabilities. The proposed SPH methodology has great potential for modelling flows when two phases are involved, e.g. solids in liquid in crystallization processes, as the flow in SPH is driven by particle-particle interaction, allowing for the 
implementation of new physics based on these interactions; successfully modelling single phase flow is an essential first step forward for multiphase cases.

\section{Acknowledgements}

The authors wish to thank the EPSRC and Heriot-Watt University for the financial support of this project and the Doctoral Training Centre in Continuous Manufacturing and Crystallization EPSRC (EP/K503289/1).

\section{References}

Adami, S., Hu, X. Y., \& Adams, N. A. (2013). A transport-velocity formulation for smoothed particle hydrodynamics. Journal of Computational Physics, 241, 292-307.

Agnew, L. R., McGlone, T., Wheatcroft, H.P., Robertson, A., Parsons, A. R., \& Wilson, C. C. (2017). Continuous crystallisation of paracetamol (acetaminophen) form II: selective access to a metastable solid form. Journal of Crystal Growth and Design, 17, 2418-2427.

Batchelor, G. K. (1974). An Introduction to Fluid Dynamics: Cambridge University Press.

Belytschko, T., Krongauz, Y., Dolbow, J., \& Gerlach, C. (1998). On the completeness of meshfree particle methods. International Journal for Numerical Methods in Engineering, 43, 785-819.

Brown, C. J., Adelakun, J. A., \& Ni, X. (2015). Characterisation and modelling of antisolvent crystallisation of salicylic acid in a continuous oscillatory baffled crystallizer. Chemical Engineering and Processing, 97, 180-186.

Brown, C. J, McGlone, T., Yerdelen, S., Srirambhatla, V., Mabbott, F., Gurung, R., L. Briuglia, M., Ahmed, B., Polyzois, H., McGinty, J., Perciballi, F., Fysikopoulos, D., MacFhionnghaile, P., Siddique, H., Raval, V., Harrington, T. S., Vassileiou, A. D., Robertson, M., Prasad, E., Johnston, A., Johnston, B., Nordon, A., Srai, J. S., Halbert, G., ter Horst, J. H., Price, C. J., Rielly, C. D., Sefcik, J., \& Florence, A. J. (2018). Enabling precision manufacturing of active pharmaceutical ingredients: workflow for seeded cooling continuous crystallisations. Molecular Systems Design \& Engineering, 3, 518-549. 
Brown, C. J., \& Ni, X.-W. (2012). Determination of metastable zone width, mean particle size and detectable number density using video imaging in an oscillatory baffled crystallizer. CrystEngComm, 14, 2944-2949.

Brown, C. J., \& Ni, X. (2011). Evaluation of growth kinetcis of antisolvent crystallisation of paracetamol in an oscillatory baffled crystalliser utilizing video imaging. Crystal Growth and Design, 11, 3994-4000.

Callahan, C. J., \& Ni, X.-W. (2012). Probing into Nucleation Mechanisms of Cooling Crystallization of Sodium Chlorate in a Stirred Tank Crystallizer and an Oscillatory Baffled Crystallizer. Crystal Growth \& Design, 12, 2525-2532.

Chew, C. M., Ristic, R. I., Reynolds, G. K., \& Ooi, R. C. (2004). Characterisation of impeller driven and oscillatory mixing by spatial and temporal shear rate distributions. Chemical Engineering Science, 59, 15571568.

Colagrossi, A., \& Landrini, M. (2003). Numerical simulation of interfacial flows by smoothed particle hydrodynamics. Journal of Computational Physics, 191, 448-475.

Courant, R., Friedrichs, K., \& Lewy, H. (1928). Über die partiellen Differenzengleichungen der mathematischen Physik. Mathematische Annalen, 100, 32-74.

Crespo, A. J. C., Gomez-Gesteira, M., \& Dalrymple, R. (2007). Boundary conditions generated by dynamic particles in SPH methods. Computers, Materials, \& Continua - Tech Science Press, 5, 173-184.

Dalrymple, R. A., \& Rogers, B. D. (2006). Numerical modeling of water waves with the SPH method. Coastal Engineering, 53, 141-147.

Deng, R., Yohanes Arifin, D., Ye Chyn, M., \& Wang, C.-H. (2010). Taylor vortex flow in presence of internal baffles. Chemical Engineering Science, 65, 4598-4605.

Dilts, G. A. (1999). Moving-least-squares-particle hydrodynamics-I. Consistency and stability. International Journal for Numerical Methodsin Engineering, 44, 1115-1155.

Eze, V. C., Fisher, J. C., Phan, A. N., \& Harvey, A. P. (2017). Intensification of carboxylic acid esterification using a solid catalyst in a mesoscale oscillatory baffled reactor platform. The Chemical Engineering Journal, 322, 205-214.

Feilden, H. (2017). CMAC Bulletin. In (5th October ed.). Glasgow, Scotland.

Ferrari, A., Dumbser, M., Toro, E. F., \& Armanini, A. (2009). A new 3D parallel SPH scheme for free surface flows. Computers \& Fluids, 38, 1203-1217.

Fitch, A. W., Jian, H., \& Ni, X. (2005). An investigation of the effect of viscosity on mixing in an oscillatory baffled column using digital 
particle image velocimetry and computational fluid dynamics simulation. Chemical Engineering Journal, 112, 197-210.

Gingold, R. A., \& Monaghan, J. J. (1977). Smoothed particle hydrodynamics: theory and application to non-spherical stars. Monthly Notices of the Royal Astronomical Society, 181, 375-389.

Gomez-Gesteira, M., Rogers, B. D., Dalrymple, R. A., \& Crespo, A. J. C. (2010). State-of-the-art of classical SPH for free-surface flows. Journal of Hydraulic Research, 48, 6-27.

González-Juárez, D., Herrero-Martín, R., \& Solano, J. P. (2018). Enhanced heat transfer and power dissipation in oscillatory-flow tubes with circular-orifice baffles: a numerical study. Applied Thermal Engineering, 141, 494-502.

González-Juárez, D., Solano, J. P., Herrero-Martín, R., \& Harvey, A. P. (2017). Residence time distribution in multiorifice baffled tubes: A numerical study. Chemical Engineering Research and Design, 118, 259-269.

Hamzah, A. A., Hasan, N., Takriff, M. S., Kamarudin, S. K., Abdullah, J., Tan, I. M., \& Sern, W. K. (2012). Effect of oscillation amplitude on velocity distributions in an oscillatory baffled column (OBC). Chemical Engineering Research and Design, 90, 1038-1044.

Howes, T., Mackley, M. R., \& Roberts, E. P. L. (1991). The simulation of chaotic mixing and dispersion for periodic flows in baffled channels. Chemical Engineering Science, $46,1669-1677$.

Howes, T., \& Shardlow, P. J. (1997). Simulation of mixing in unsteady flow through a periodically obstructed channel. Chemical Engineering Science, 52, 1215-1225.

Jian, H. (2002). PhD thesis: Understanding unsteadiness and turbulence in two chemical engineering systems. Heriot-Watt University, Edinburgh, Scotland.

Jian, H., Fitch, A. W., \& Ni, X. (2004). Numerical and experimental investigations into the effect of gap between baffle and wall on mixing in an oscillatory baffled column. International Journal of Chemical Reactor Engineering, 2, 1-16.

Jian, H., \& Ni, X.-W. (2003). On modelling turbulent flow in an oscillatory baffled column - RANS model or large-eddy simulation? Journal of Chemical Technology \& Biotechnology, 78, 321-325.

Jian, $\mathrm{H}$., \& Ni, X. (2005). A numerical study on the scale-up behaviour in oscillatory baffled columns. Chemical Engineering Research and Design, 83, 1163-1170.

Kimuli, E. N., Onyemelukwe, I. I., Benyahia, B., \& Rielly, C. D. (2017). Characterisation of axial dispersion in a Meso-scale Oscillatory Baffled Crystalliser using a Numerical Approach. In A. Espuña, M. 
Graells \& L. Puigjaner (Eds.), Computer Aided Chemical Engineering (Vol. 40, pp. 223-228): Elsevier.

Lawton, S., Steele, G., Shering, P., Zhao, L., Laird, I., \& Ni, X.-W. (2009). Continuous Crystallization of Pharmaceuticals Using a Continuous Oscillatory Baffled Crystallizer. Organic Process Research \& Development, 13, 1357-1363.

Lee, E. S., University of Manchester. School of Mechanical, A., \& Engineering, C. (2007). Truly Incompressible Approach for Computing Incompressible Flow in SPH and Comparisons with the Traditional Weakly Compressible Approach: University of Manchester.

Mackley, M. R., \& Neves Saraiva, R. M. C. (1999). The quantitative description of fluid mixing using Lagrangian- and concentrationbased numerical approaches. Chemical Engineering Science, 54, 159170.

Mackley, M. R., \& Ni, X. (1991). Mixing and dispersion in a baffled tube for steady laminar and pulsatile flow. Chemical Engineering Science, 46, 3139-3151.

Mackley, M. R., \& Ni, X. (1993). Experimental fluid dispersion measurements in periodic baffled tube arrays. Chemical Engineering Science, 48, 3293-3305.

Mackley, M. R., \& Roberts, E. P. L. (1991). Mixing and flow patterns for unsteady flow in baffled channels. Generals Papers in Fluid Engineering AIChE., 127, 57-64.

Manninen, M., Gorshkova, E., Immonen, K., \& Ni, X.-W. (2013). Evaluation of axial dispersion and mixing performance in oscillatory baffled reactors using CFD. Journal of Chemical Technology \& Biotechnology, 88, 553-562.

Mazubert, A., Fletcher, D. F., Poux, M., \& Aubin, J. (2016a). Hydrodynamics and mixing in continuous oscillatory flow reactors-Part I: Effect of baffle geometry. Chemical Engineering and Processing: Process Intensification, 108, 78-92.

Mazubert, A., Fletcher, D. F., Poux, M., \& Aubin, J. (2016b). Hydrodynamics and mixing in continuous oscillatory flow reactors-Part II: Characterisation methods. Chemical Engineering and Processing: Process Intensification, 102, 102-116.

Monaghan, J. J. (1992). Smoothed Particle Hydrodynamics. Annual Review of Astronomy and Astrophysics, 30, 543-574.

Monaghan, J. J. (1994). Simulating Free Surface Flows with SPH. Journal of Computational Physics, 110, 399-406.

Morris, J. P. (1996). A Study of the Stability Properties of Smooth Particle Hydrodynamics. Publications of the Astronomical Society of Australia, 13, 97-102. 
Morris, J. P., Fox, P. J., \& Zhu, Y. (1997). Modeling Low Reynolds Number Incompressible Flows Using SPH. Journal of Computational Physics, 136, 214-226.

Navarro Fuentes, F., Keane, M. A., \& Ni, X. (2018). A comparative evaluation of a multiphase catalytic hydrogenation in an oscillatory baffled reactor and a stirred tank reactor. Organic Research Process and Development, Submitted.

$\mathrm{Ni}, \mathrm{X}$. (1994). Residence time distribution measurements in a pulsed baffled tube bundle. Journal of Chemical Technology \& Biotechnology, 59, 213-221.

$\mathrm{Ni}, \mathrm{X}$. (1995). A study of fluid dispersion in oscillatory flow through a baffled tube. Journal of Chemical Technology \& Biotechnology, 64, 165-174.

Ni, X., Jian, H., \& Fitch, A. (2003). Evaluation of Turbulent Integral Length Scale in an Oscillatory Baffled Column Using Large Eddy Simulation and Digital Particle Image Velocimetry. Chemical Engineering Research and Design, 81, 842-853.

Ni, X., Jian, H., \& Fitch, A. W. (2002). Computational fluid dynamic modelling of flow patterns in an oscillatory baffled column. Chemical Engineering Science, 57, 2849-2862.

Ni, X., \& Stevenson, C. C. (1999). On the effect of gap size between baffle outer diameter and tube inner diameter on the mixing characteristics in an oscillatory-baffled column. Journal of Chemical Technology \& Biotechnology, 74, 587-593.

Ni, X., Valentine, A., Liao, A., Sermage, S. B. C., Thomson, G. B., \& Roberts, K. J. (2004). On the Crystal Polymorphic Forms of l-Glutamic Acid Following Temperature Programmed Crystallization in a Batch Oscillatory Baffled Crystallizer. Crystal Growth \& Design, 4, 11291135.

Ni, X., Zhang, Y, \& Mustafa, I. (1999). Correction of Polymer Particle Size with Droplet Size in Suspension Polymerisation of Methylmethacrylate in a Batch Oscillatory Baffled Reactor. Chemical Engineering Science, 54, 841-850.

Nogueira, X., Taylor, B. J., Gomez, H., Colominas, I., \& Mackley, M. R. (2013). Experimental and computational modeling of oscillatory flow within a baffled tube containing periodic-tri-orifice baffle geometries. Computers \& Chemical Engineering, 49, 1-17.

Ottino, J. M. (1989). The Kinematics of Mixing: Stretching, Chaos, and Transport: Cambridge University Press.

Palma, M., \& Giudici, R. (2003). Analysis of axial dispersion in an oscillatory-flow continuous reactor. Chemical Engineering Journal, 94, 189-198. 
Panizzo, A. (2004). PhD thesis: Physical and numerical modelling of subaerial landslide generated waves. Universita degli Studi di L'Aquila.

Phan, A. N., \& Harvey, A. (2010). Development and evaluation of novel designs of continuous mesoscale oscillatory baffled reactors. Chemical Engineering Journal, 159, 212-219.

Phan, A. N., \& Harvey, A. P. (2011). Effect of geometrical parameters on fluid mixing in novel mesoscale oscillatory helical baffled designs. Chemical Engineering Journal, 169, 339-347.

Phan, A. N., Harvey, A. P., \& Eze, V. (2012). Rapid production of biodiesel in mesoscale oscillatory baffled reactors. Chemical Engineering and Technology, 35, 1214-1220.

Reis, N., Harvey, A. P., Mackley, M. R., Vicente, A. A., \& Teixeira,J. A. (2005). Fluid mechanics and design aspects of a novel oscillatory flow screening mesoreactor. Chemical Engineering Research and Design, 83, 357-371.

Reis, N., Vicente, A. A., \& Teixeira, J. A. (2010), Liquid backmixing in oscillatory flow through a periodically constricted meso-tube. Chemical Engineering and Processing: Process Intensification, 49, 793-803.

Reis, N., Vicente, A. A., Teixeira, J. A., \& Mackley, M. R. (2004). Residence times and mixing of a novel continuous oscillatory flow screening reactor. Chemical Engineering Science, 59, 4967-4974.

Roberts, E. P. L. (1994). A numerical and experimental study of transition processes in an obstructed channel flow. Journal of Fluid Mechanics, 260, 185-209.

Roberts, E. P. L., \& Mackley, M. R. (1995). The simulation of stretch rates for the quantitative prediction and mapping of mixing within a channel flow. Chemical Engineering Science, 50, 3727-3746.

Roberts, E. P. L, \& Mackley, M. R. (1996). The development of asymmetry and period doubling for oscillatory flow in baffled channels. Journal of Fluid Mechanics, 328, 19-48.

Su, Q., Benyahia, B., Nagy, Z. K., \& Rielly, C. D. (2015). Mathematical modeling, design, and optimization of a multi-segment multi-addition plug-flow crystallizer for anti-solvent crystallizations. Organic Process Research \& Development, 19, 1859-1870.

Verlet, L. (1967). Computer "Experiments" on Classical Fluids. I. Thermodynamical Properties of Lennard-Jones Molecules. Physical Review, 159, 98-103.

Violeau, D. (2012). Fluid mechanics and the SPH method : theory and applications. Oxford: Oxford University Press. 
Wendland, H. (1995). Piecewise polynomial, positive definite and compactly supported radial functions of minimal degree. Adv. Comput. Math., 4, 389-396.

Wilson, B., Ni, X., \& Sherrington, D. C. (2001). On the investigation of phasetransfer catalysis reaction in an oscillatory baffled reactor. Journal of Industrial and Engineering Chemistry Research, 40, 5300-5304.

Yang, C., \& Mao, Z.-S. (2014). Chapter 6 - Crystallizers: CFD-PBE modeling. In Numerical Simulation of Multiphase Reactors with Continuous Liquid Phase (pp. 263-294). Oxford: Academic Press.

Zhang, C., Hu, X. Y., \& Adams, N. A. (2017). A weakly compressible SPH method based on a low-dissipation Riemann solver. Journal of Computational Physics, 335, 605-620.

Zheng, M., Li, J., Mackley, M. R., \& Tao, J. (2007). The development of asymmetry for oscillatory flow within a tube containing sharp edge periodic baffles. Physics of Fluids, 19, 114101.

Zheng, M., \& Mackley, M. (2008). The axial dispersion performance of an oscillatory flow meso-reactor with relevance to continuous flow operation. Chemical Engineering Science, 63, 1788-1799. 